.

\title{
Optimized survey design for Electrical Resistivity Tomography: combined optimization of measurement configuration and electrode placement
}

\author{
Sebastian Uhlemann ${ }^{1,2, *}$, Paul B. Wilkinson ${ }^{1}$, Hansruedi Maurer ${ }^{2}$, Florian M. Wagner ${ }^{3}$, \\ Timothy C. Johnson ${ }^{4}$, and Jonathan E. Chambers ${ }^{1}$ \\ 1 - British Geological Survey, Environmental Science Centre, Keyworth, Nottingham, UK \\ 2 - ETH Zurich, Institute of Geophysics, Zurich, Switzerland \\ 3 - University of Bonn, Steinmann Institute, Department of Geophysics, Bonn, Germany \\ 4 - Pacific Northwest National Laboratory, Richland, USA
}

Date Submitted: 22/11/2017

Date Revision Submitted: 21/03/2018

Abbreviated title: Optimization of ERT measurement configuration and electrode placement

(1)

2


33 Within geoelectrical imaging, the choice of measurement configurations and electrode 34 locations is known to control the image resolution. Previous work has shown that optimized 35 survey designs can provide a model resolution that is superior to standard survey designs. This paper demonstrates a methodology to optimize resolution within a target area, while limiting the number of required electrodes, thereby selecting optimal electrode locations. This is achieved by extending previous work on the 'Compare-R' algorithm, which by calculating updates to the resolution matrix optimizes the model resolution in a target area. Here, an additional weighting factor is introduced that allows to preferentially adding measurement configurations that can be acquired on a given set of electrodes. The performance of the optimization is tested on two synthetic examples and verified with a laboratory study. The effect of the weighting factor is investigated using an acquisition layout comprising a single line of electrodes. The results show that an increasing weight decreases the area of improved resolution, but leads to a smaller number of electrode positions. Imaging results superior to a standard survey design were achieved using $56 \%$ fewer electrodes. The performance was also tested on a 3D acquisition grid, where superior resolution within a target at the base of an embankment was achieved using $22 \%$ fewer electrodes than a comparable standard survey. The effect of the underlying resistivity distribution on the performance of the optimization was investigated and it was shown that even strong resistivity contrasts only have minor impact. The synthetic results were verified in a laboratory tank experiment, where notable image improvements were achieved. This work shows that optimized surveys can be designed that have a resolution superior to standard survey designs, while requiring significantly fewer electrodes. This methodology thereby provides a means for improving the efficiency of geoelectrical imaging.

57 Keywords: Hydrogeophysics, Inverse Theory, Electrical Resistivity Tomography, Electrical 58 Properties 
60

61

62

Within the last two decades geoelectrical data acquisition and processing have seen crucial developments. Automatic, multi-channel measurement systems combined with autonomous processing schemes nowadays allow for real-time electrical resistivity tomography (ERT) monitoring (Johnson, 2016; Parsekian et al., 2015; Singha et al., 2014). This has opened the opportunity to study a wide variety of subsurface processes, such as nuclear waste decommissioning (Daily et al., 2004; Johnson et al., 2012; Kuras et al., 2016), $\mathrm{CO}_{2}$ sequestration (Benisch et al., 2015; Schmidt-Hattenberger et al., 2016), landslide hydrology (Gance et al., 2016; Supper et al., 2014; Uhlemann et al., 2017), permafrost degradation (Hilbich et al., 2008; Krautblatter et al., 2010), and landfill processes (Dumont, 2017; Godio et al., 2015; Grellier et al., 2008). Monitoring studies where data are acquired on hundreds of electrodes within short timescales are becoming more frequent (Kuras et al., 2016; Uhlemann et al., 2017). Nevertheless, the time required for data acquisition, and how to handle and interpret the vast amount of data such installations provide are posing new challenges (Parsekian et al., 2015; Rucker, 2014). To overcome these, efforts are undertaken to limit the amount of data without reducing their information content.

This can be achieved by optimizing the survey design, which can be broadly divided into two approaches. The most common approach is to take a set of electrodes and choose measurement combinations from it that maximises the image resolution (Loke et al., 2013). Those algorithms can achieve an image resolution superior or equal to standard survey designs, e.g. WennerSchlumberger or dipole-dipole, with the same or fewer number of measurements (Loke et al., 2014; Stummer et al., 2004; Wilkinson et al., 2012). The other approach is to optimize the sensor positions. Wagner et al. (2015) showed that by using an optimized set of electrode locations the resolution within a target horizon can be significantly improved compared to 
conventional equally spaced electrode arrays. Both approaches reduce the amount of data while

84

85 preserving image resolution.

Here we present a novel algorithm that combines these two approaches. We extend the methodology introduced by Wilkinson et al. (2015), which optimized measurement configurations to improve image resolution within a target area, by preferentially adding measurement configurations that can be acquired on a given set of electrodes. The new approach is tested on a synthetic example, where measurement configurations and electrode positions are chosen from a linear electrode array, and by a laboratory experiment simulating a 3D measurement setup (i.e. an electrode grid) on an embankment. We show that this methodology can generate an optimal set of electrode locations and measurement configurations that is a fraction of all possible locations and configurations, while still offering equal or superior resolution to standard survey designs. This is in contrast to previous optimisation strategies that solely aimed to improve the model resolution (Loke et al., 2013; Stummer et al., 2004; Wilkinson et al., 2015). The presented approach will not only aid in creating survey designs for optimal resolution of a target area, but also reduce costs for ERT installations, as fewer electrodes and cables will be required for the optimized survey. Hence it addresses exactly what Curtis and Maurer (2000) define as an optimal survey, i.e., a survey that leads to high accuracy and reliability of the model estimates, while being easily realizable under minimal financial effort. Thus, this methodology will aid in improving the efficiency of ERT data acquisition, in particular if a priori information about the subsurface is available. While it is applied to an ERT example here, the approach should be easily transferrable to optimizing image resolution for other geophysical tomographic methods.

\section{METHODOLOGY}


Most recent studies on ERT measurement optimization make use of the model resolution matrix (Alfouzan et al., 2010; Loke et al., 2015b, 2014, 2010; Stummer et al., 2004; Wagner et al., 2015; Wilkinson et al., 2015, 2012, 2006). In comparison to sensitivity based optimization strategies (Athanasiou et al., 2009; Tsakirrbaloglou et al., 2016; Tsourlos et al., 2016), the model resolution accounts for linear (in)dependency between measurement configurations, and is therefore used here as well. The model resolution matrix $\mathbf{R}$ quantifies how well each model cell of a resistivity image is resolved by the measured data. For the linearized iterative GaussNewton solution of the ERT problem, $\mathbf{R}$ is defined as (Wilkinson et al., 2006):

$$
\mathbf{R}=\left(\mathbf{G}^{\mathrm{T}} \mathbf{G}+\mathbf{C}\right)^{-1} \mathbf{G}^{\mathrm{T}} \mathbf{G},
$$

115

with the Jacobian matrix $\mathbf{G}$ and the constraint matrix $\mathbf{C}$. The main diagonal elements $R_{j}$ of $\mathbf{R}$ are referred to here as the model resolution and range between 0 and 1 , where $R_{j}=0$ represents an entirely unresolved, and $R_{j}=1$ a perfectly resolved cell $j$. Although $\mathbf{C}$ could represent any kind of model constraints (Loke et al., 2014; Wilkinson et al., 2012), here it is defined as $\mathbf{C}=$ $\lambda \mathbf{I}$, with $\mathbf{I}$ being the identity matrix, to represent a simple damped least square problem (Wilkinson et al., 2006). The choice of the damping factor $\lambda$ is problem specific, with larger values leading to lower resolution (Loke et al., 2010). For this type of optimization problem, $\lambda$ is often chosen so that the model resolution is small $(R \approx 0.05)$ at a certain distance from the electrodes, typically at the base of the model (Stummer et al., 2004; Wilkinson et al., 2006). Note that $\lambda$ is not only affecting the absolute values of the diagonal elements of $\mathbf{R}$, but also the distribution of the relative magnitudes. Nevertheless, Loke et al. (2010) have shown that the relative performance of the optimization is not particularly sensitive to the value of $\lambda$.

The optimization is an iterative process and starts from a small set of measurements from a small number of electrodes. Additional measurements are selected from a comprehensive set, comprising alpha and beta-type configurations (Loke et al., 2015). For each possible new 
measurement, the change in the resolution matrix $\Delta \mathbf{R}$ is calculated using a Sherman-Morrison Rank-1 update of the resolution matrix, which is defined as (Loke et al., 2014; Wilkinson et al., 2006):

$$
\Delta \mathbf{R}_{\mathbf{b}}=\frac{\mathbf{z}}{1+(\mathbf{g} \cdot \mathbf{z})}\left(\mathbf{g}^{\mathrm{T}}-\mathbf{y}^{\mathrm{T}}\right)
$$

where

$$
\mathbf{z}=\left(\mathbf{G}_{\mathrm{b}}^{\mathrm{T}} \mathbf{G}_{\mathrm{b}}+\mathbf{C}\right)^{-1} \mathbf{g}, \mathbf{y}=\left(\mathbf{G}_{\mathrm{b}}^{\mathrm{T}} \mathbf{G}_{\mathrm{b}}\right) \mathbf{z}
$$

with the Jacobian matrix $\mathbf{G}$ b consisting of the sensitivities of the measurements of the current base set, and $\mathbf{g}$ comprising the sensitivities of the new test configuration. Following Wilkinson et al. (2015) all additional measurements are ranked according to the calculated improvement of the resolution in the target region

$$
F_{\mathrm{CR}}=\frac{1}{m} \sum_{j=1}^{m} \frac{w_{\mathrm{t}, j} \Delta R_{\mathrm{b}, j}}{R_{\mathrm{c}, j}}
$$

with the number of model cells $m$, the resolution of cell $j$ given by the comprehensive set $R_{c, j}$, and a weighting factor $w_{t, j}$ that is 1 if cell $j$ is within the target region and $10^{-12}$ if not. Survey designs are often "focused" on specific target areas, which requires a priori information about the subsurface properties (Curtis, 1999; Furman et al., 2007; Loke et al., 2015b; Roux and Garcia, 2014). In order to penalize measurements that would require electrodes other than those already present in the current base set, the weighting factor we was added to equation 4 :

$$
F_{\mathrm{CR}}=\frac{1}{m w_{\mathrm{e}}^{\beta}} \sum_{j=1}^{m} \frac{w_{\mathrm{t}, j} \Delta R_{\mathrm{b}, j}}{R_{\mathrm{c}, j}}
$$

For a given measurement, $w_{\mathrm{e}}=\left(1+n_{\mathrm{e}}\right)$ where $n_{\mathrm{e}}$ is the number of additional electrodes required (from 0 to 4). This weighting factor is controlled by the exponent $\beta$; increasing values of $\beta$ 
cause a stronger penalty for measurements requiring additional electrodes. The highest ranked

151

152

153

154

155

156

157 measurement is added to the current base set. The second highest is only added if its linear dependency to the first is below a certain limit. Wilkinson et al. (2012) showed that superior results can be obtained by setting this limit to the value of the current average relative resolution $S$, defined as

$$
S=\frac{1}{n} \sum_{k=1}^{n} \frac{R_{\mathrm{b}, k}}{R_{\mathrm{c}, k}}
$$

which was evaluated for all cells $k$ within the target volume. Linearity tests are performed and measurements added until a certain fraction of the size of the current base set have been added, defined by the step size of the iterative optimization process. After each iteration, $\mathbf{R}_{\mathrm{b}}$ is recalculated. Loke et al. (2014) showed that the performance of the optimization degrades with increasing step size, however, computationally larger step sizes are preferable as $\mathbf{R}_{\mathrm{b}}$ needs to be recalculated fewer times; this is further discussed in the following section.

Calculations of $\mathbf{G}, \mathbf{R}_{\mathrm{c}}, \mathbf{R}_{\mathrm{b}}$, and $F_{\mathrm{CR}}$ were facilitated by adapting the fully parallelized source code of E4D (Johnson et al., 2010), and exploiting OpenBLAS routines (Wang et al., 2013) to improve computational performance of the $\mathbf{R}$ and $F_{C R}$ calculations. All optimizations presented in this study were calculated on a machine with four Intel ${ }^{\circledR} \mathrm{Xeon}^{\circledR}$ E5-2697V3 CPUs, comprising in total 56 cores running at $2.6 \mathrm{GHz}$. Loke et al. (2015) found that using single precision, compared to double precision, caused only a marginal change in the calculated model resolution, while significantly reducing calculation times. Hence, single precision was used in the calculation of $\mathbf{R}$ and $F_{\mathrm{CR}}$.

For $N$ electrodes $N(N-1)(N-2)(N-3) / 8$ unique four-point measurements can theoretically be acquired (accounting for polarity and reciprocity); for 32 and 117 electrodes this would equal 107880 and 22241115 measurements, respectively. Evaluating all of these measurements 
would be computationally very demanding and some measurements would be impractical to acquire due to small signal-to-noise ratios and high sensitivities to electrode misplacements. Thus, the comprehensive set from which measurements are added at each iteration comprises only alpha and beta-type configurations that have geometric factors and sensitivities below specified problem-specific limits.

\section{LINEAR ELECTRODE ARRAY}

The methodology was tested first on a simple synthetic model, comprising 32 possible electrode locations spaced by $1 \mathrm{~m}$ along a single line. A trapezoidal prism in the centre of the model formed the target volume, within which the resolution was to be optimized (Figure 1). As the methodology was developed for 3D problems, this example was calculated on a 3D representation of a linear electrode array. The model domain was discretized using an unstructured tetrahedral mesh, comprising 3312 elements (equal to the number of model parameters $m$ ), which was refined around the electrode locations and extended beyond to account for 'outer-space' sensitivities (Maurer and Friedel, 2006). The comprehensive set comprised alpha and beta-type configurations with a maximum geometric factor $K_{\max }=4146.9$ $\mathrm{m}$ (equal to a dipole-dipole geometric factor for $a=1$ and $n=10$ ) and a maximum geometric sensitivity of $s / K=5 \mathrm{~m}^{-1}$ (Wilkinson et al., 2008), totalling 70555 four-point measurement configurations. A description of the alpha and beta-type arrays can be found in Loke et al. (2015a). Szalai and Szarka (2011) present other possible measurement configurations that could be added to the comprehensive set (e.g. "Null" or "Quasi-null" arrays). However, some of those may cause instabilities in the inversion if the data and model parameters are logarithmically transformed (Johnson et al., 2010), which is desirable due to the large range of resistivities often encountered in geoelectrical imaging. Measurements involving remote electrodes (pole-pole or pole-dipole) could also be included, but often present difficulties in practical site investigations and cannot be used in tank experiments. Restricting the 
comprehensive set to alpha- and beta-type configurations below a certain limit for their geometric factor removes measurement configurations that are likely to be unstable (Loke et al., 2014). The damping factor $\lambda=0.004$ was chosen so that the model resolution was small at the base of the model $(R<0.05$; Wilkinson et al., 2006). The initial measurement set comprised 30 measurements employing six electrodes located above the target area.

To investigate the effect of the exponent $\beta$, which controls the penalty for including additional electrodes at every iteration, the optimization was run for values of $\beta=0.0,2.0$, and 5.0 (Figure 2). Each optimization employed a step size of $5 \%$, meaning that at each iteration the number of measurements in the optimized set increased by $5 \%$. Setting $\beta=0.0$ is equivalent to the methodology introduced by Wilkinson et al. (2015) to optimize resolution within a target region. For $\beta=0.0$ all possible electrode locations are used once the set includes more than 327 measurements, which is reached within the first 49 iterations. This 'unconstrained' optimization yields mostly superior resolution compared to employing larger values of $\beta$. For $\beta=2.0$ all possible electrodes are included in the survey once it comprises 6201 measurements. From this point, the resolution achieved in the optimization is independent of $\beta$. Despite using up to $59 \%$ fewer electrodes, the relative resolution achieved with $\beta=2.0$ is similar to $\beta=0.0$, with differences in average relative resolution being less than 0.06 for all iterations. During a few iterations, i.e., between 386 and 1303 measurements (Figure 2b), the relative resolution obtained with $\beta=2.0$ is superior to $\beta=0.0$, with a maximum difference of 0.016 . This is likely to be an effect of a localised optimum that was found by constraining the optimization to use a certain set of electrodes. However, once the measurement set comprises more than 1303 measurements, the constraints on adding additional electrodes limit the increase in relative resolution compared to $\beta=0.0$.

221 Using $\beta=5.0$, for small measurement sets the optimized survey employs considerably fewer electrodes than $\beta=0.0$ or 2.0 . When the survey comprises about 2750 measurements, $\beta=0.0$ 
uses all 32 electrodes and $\beta=2.0$ uses 23 electrodes, while $\beta=5.0$ uses only 17 electrodes, thus only $53 \%$ of the available electrodes. This, however, also results in a relative resolution 0.22 smaller than for $\beta=0.0$. For less than 1500 measurements (Figure 2b), this difference is smaller than 0.09 , despite using about $50 \%$ fewer electrodes than $\beta=0.0$. The $\beta=5.0$ optimized survey includes all possible electrodes once the set comprises more than 15000 measurements. In general, the higher $\beta$ the longer a certain set of electrodes is used to optimize the resolution, leading to a decreasing performance of the optimization.

Figure 2 also shows the relative resolution of a standard survey, comprising 934 dipole-dipole and Wenner-Schlumberger measurements and using all 32 electrodes. This shows the benefit of the presented approach clearly. The optimization, for all tested values of $\beta$, achieves a relative resolution in the target area higher than the standard survey $(S=0.185)$. For $\beta=0.0$, the improvement in the relative resolution is 0.042 , for $\beta=2.0$ it is 0.054 and for $\beta=5.0$ it is 0.018. In the case of $\beta=2.0$ and $\beta=5.0$ this improvement is achieved using $43.8 \%$ and $56.3 \%$ fewer electrodes than used in the standard survey, respectively.

Figure 3 shows the resolution within the imaging plane for the standard and optimized surveys, employing 934 measurements each, and the difference in resolution between the optimized and standard survey. The resolution of the standard survey shows the usual distribution with high resolution close to the electrodes, and a fast decline with increasing distance from the electrodes. Within the target area a similar behaviour can be found; the upper part is perfectly resolved, while the lower part exhibits a resolution $<0.3$. The optimization is set to improve the resolution within this target area. All tested values of $\beta$ gain a higher resolution than the standard survey in this part of the imaging plane, and image more than half of the target area with a resolution $>0.9$. While for $\beta=0.0$, the entire imaging plane shows high resolution, especially close to the surface, and increases with depth, for higher $\beta$ values high resolution is only achieved close to the target area. The higher $\beta$ the fewer electrodes are used and the smaller 
the well-resolved area becomes. The difference between optimized and standard resolution highlights this behaviour (Figure $3 \mathrm{e}-\mathrm{g}$ ). While for $\beta=0.0$ the resolution in the target area improves by more than 0.5 , which is an increase of more than $100 \%$, considerable improvements are also achieved in nearly the entire imaging plane, except in areas close to the surface towards the boundaries, where the resolution is slightly smaller than for the standard survey. In the target area, $\beta=2.0$ provides comparable increases in resolution to $\beta=0.0$, improvements of up to 0.35 are gained using $\beta=5.0$. Outside the target, the area with improved resolution decreases with increasing $\beta$, and areas with worse resolution increase. The parts of the imaging plane with decreased resolution are linked to the smaller set of electrodes used. In more uncertain then smaller values of $\beta$ should be used.

The impact of the step size on the performance of the optimization was also investigated; Figure 4 shows the performance for step sizes of $2 \%, 5 \%$, and $10 \%$. The main difference is when additional electrodes are added to the survey during the optimization. Generally, step sizes of $5 \%$ and $10 \%$ tend to add more electrodes at a single iteration than added when using a step size of $2 \%$. This is particularly evident when the optimized set comprises about 5100 measurements. At step sizes of $10 \%$ and $5 \%$, ten and seven electrodes are added, respectively, while for $2 \%$ only one additional electrode is used. Those differences in the use of electrodes also cause the differences in relative resolution obtained by the different step sizes. The effect is comparably small for optimized sets comprising less than 5500 measurements, but becomes more significant for larger measurement sets, where differences in the relative resolution reach 0.11 . This larger difference is caused by a $2 \%$ step size using $13.8 \%$ fewer electrodes than employed for a step size of $10 \%$. Figure $4 \mathrm{~b}$ shows that where the same number of electrodes are used, 
regardless of step size, the optimized relative resolution is virtually identical. With the increasing number of iterations, the calculation times increase considerably; while a $10 \%$ step size was calculated in $7.4 \mathrm{~h}, 5 \%$ took $15.4 \mathrm{~h}$, and $2 \% 26.3 \mathrm{~h}$.

The actual imaging performance of the survey designs is shown in Figure 5, where inverted resistivity models are presented. In the forward model (Figure 5a), the area for which the resolution was optimized was given a resistivity of $10 \Omega \mathrm{m}$, while the background had a resistivity of $100 \Omega \mathrm{m}$. The forward problem was implemented and solved in Res3DMod (Geotomo Software, Malaysia). The synthetic data were contaminated with voltage-dependent noise defined as:

$$
|e|=a+b\left|R_{t}\right|
$$

with $a=0.05 \Omega$, and $b=0.02$.

All models were inverted using an L1 norm on the model roughness, and the data were fitted to their respective errors $\left(\chi^{2}=1.0\right)$. The comprehensive set, forming the benchmark for this comparison, is able to delineate the target in its correct position and approximate extent; the shape can be recognized in the inverted resistivity model, but is considerably smoothed and imaged with a higher vertical extent. The target area is imaged with a minimum resistivity of $34.4 \Omega \mathrm{m}$ and a mean of $52.3 \Omega \mathrm{m}$, while the background has a mean resistivity of $95.1 \Omega \mathrm{m}$. The standard survey, employing only 934 measurements (1.3\% of the comprehensive set) and all 32 possible electrode location, fails in imaging the true shape and depth of the target. It is imaged as a subvertical feature with a mean resistivity of $53.4 \Omega \mathrm{m}$ in the true target location, thus $1.1 \Omega \mathrm{m}$ higher than imaged by the comprehensive survey. The background resistivity has a mean of $96.8 \Omega \mathrm{m}$. The optimized set images the target in a shape similar to the comprehensive set and with a mean resistivity of $45.2 \Omega \mathrm{m}$ and a minimum of $24.1 \Omega \mathrm{m}$, thus closer to the true resistivity model than imaged by the standard survey. The background is imaged at a mean 
resistivity of $80.7 \Omega \mathrm{m}$, and thus lower than for the comprehensive and standard surveys. This is an effect of the lower resolution outside the target area. The uncentered Pearson $r$ correlation coefficient of the target area showed a stronger correlation between the true resistivity model and optimized inversion result $\left(r_{\mathrm{opt}}=0.89\right)$ than between the true resistivity model and standard survey results $\left(r_{\text {standard }}=0.78\right)$. Similarly, the root-mean-squared $(\mathrm{RMS})$ difference between the true resistivity model and the results of the optimized survey was $32.7 \Omega \mathrm{m}$, while it was 43.7 $\Omega \mathrm{m}$ for the standard survey. Outside the target area is where the optimized set performs worse than the standard survey. Thus, within the target horizon the optimized set images the true the target.

Both the spatial distribution of possible electrode locations and the electrodes comprising the initial set are variables affecting the results of the optimization. To show this, we recalculated the optimization using $\beta=5.0$ but employing a different initial set of electrodes, with four electrodes being placed close to the model boundaries, and three close to the target (white dots in Figure 6, initial set B). The results are similar to what was achieved with the first initial set comprising electrode locations directly over the target (initial set A). The resolution within the target is virtually identical (compare Figures $3 \mathrm{~d}$ and $6 \mathrm{a}$ ), but more resolution is retained in standard survey (Figures $3 \mathrm{~g}$ and $6 \mathrm{~b}$ ). In both cases, the optimized survey shows increased resolution in lower parts of the target area, which extend outside the target boundaries. Using initial set B this area outside the target is smaller than for initial set A. The inverted resistivity model shows the target with a similar shape to that imaged using the optimized survey of initial set A, but with a shape more comparable to the results obtained by the comprehensive survey. 
322 This is an effect of a higher fraction of measurements with low geometric factor in the optimized survey of initial set B. These measurements have a smaller error and thus lead to a better imaging performance. The additional use of measurements close to the model boundaries cause a lower reduction of resolution in this area and improved the recovery of the true resistivity in these parts of the model. Despite the different initial sets of electrodes, the performance of the optimizations are comparable in both the achieved resolution of the target and used electrode locations.

\section{3D MEASUREMENT GRID}

Extending surveys into three dimensions usually leads to significant increases in the number of electrode locations and measurements. Hence, this is where the proposed optimization methodology is expected to show the greatest benefits. In order to test the optimization, a 3D synthetic example was designed, comprising 117 electrodes, arranged in a grid of 13 electrodes along the $x$-axis, and 9 electrodes along the $y$-axis. The setup replicates a typical embankment situation, e.g. a flood embankment or mining tailings dam, where electrodes are deployed only on one side (Figure 7). To resemble typical conditions, the "embankment" has a 1 in 2 slope (Glendinning et al., 2014), miniaturized into an assumed laboratory tank set-up being $1 \mathrm{~m}$ long and wide, with an embankment height of $0.25 \mathrm{~m}$. The results of this synthetic study are used in the following laboratory study, testing the methodology on real data. The electrode spacing in $x$-direction was chosen to be $0.075 \mathrm{~m}$, and $0.1 \mathrm{~m}$ in the $y$-direction. The impact of different model mesh sizes on the performance of the optimization was tested and showed only negligible effects. Thus, a relatively coarse discretization using 9711 tetrahedral elements was used. The target was defined as a rectangular prism, with dimensions of $0.68 \mathrm{~m}, 0.3 \mathrm{~m}$, and $0.06 \mathrm{~m}$ along the $x, y$, and $z$-directions. It was placed centrally at the base of the slope, resembling an area that could potentially be affected by soil piping or a different failure type 
at the base of an embankment. Neumann-boundary conditions were used at the outer and lower model boundaries to account for the insulating tank walls. The comprehensive set comprised a subset of alpha and beta configuration, including inline, crossline, and diagonal alpha and betatype configurations, as well as equatorial beta-type configurations (sensitivity patterns of these measurement types are discussed in detail in Loke et al., 2014), with a maximum geometric factor of $K_{\max }=345 \mathrm{~m}$ (equal to a dipole-dipole geometric factor for $a=1$ and $n=10$ ) and a geometric sensitivity of $s / K=100 \mathrm{~m}^{-1}$. The grid of electrodes extended close to the model boundaries, which were found to have a significant impact on the calculation of the geometric factor. Thus, the comprehensive set was filtered on the geometric factors calculated using a homogeneous forward model incorporating the correct boundary conditions (Loke et al., 2014); after filtering it included 12755 measurements. The damping factor $\lambda=0.05$ was chosen so that the resolution at the base of the model was $R<0.05$. The computation time of this optimization was $10.2 \mathrm{~h}$.

The initial set for the optimization comprised six electrodes, located centrally above the target volume, and 20 measurements. Considering the optimization performance obtained on the linear electrode array example, the 3D optimization was run using a step size of $10 \%$ and for a weighting exponent of $\beta=5.0$ (Figure 8). Optimization studies often assume a homogeneous model resistivity for generality, and previous studies have shown that moderate deviations from could have a potential impact on the optimization as potential fields are considerably disturbed. Thus, rather than testing the optimization performance regarding $\beta$ and the step size, the effects of the underlying resistivity model are investigated. Hence, optimized survey designs were calculated assuming a homogeneous resistivity model of $10 \Omega \mathrm{m}$, and a resistivity model with a resistive target $(5000 \Omega \mathrm{m})$ in a $10 \Omega \mathrm{m}$ background medium. As a real data application is 
considered, measurement errors are incorporated into the optimization (Wilkinson et al., 2012) and assumed to be a function of the transfer resistance $R_{t}$ (eq. 7).

Figure 7 shows the resolution of the comprehensive set for both situations. While for the homogeneous model (Figure 7a) the target is well resolved $(R>0.5)$ between $x=0.4$ and $x=$ $0.6 \mathrm{~m}$, defining it as a resistive feature lowers the resolution within the target significantly $(R$ $<0.05)$. This is because current will flow predominantly through the background medium and not through the highly resistive target volume.

This considerable change in the comprehensive set resolution affects the performance of the optimization (Figure 8), as different measurements will need to be chosen to resolve a resistive target, compared to a target with a similar resistivity to the background medium. Generally, the lower the resolution in the resistive target, the lower the relative resolution that is achieved at a given number of measurements, compared to the optimization using a homogeneous resistivity model; the largest difference $\Delta S=0.31$ occurs with the optimized set comprising 3345 measurements. Although, the absolute improvement is smaller for the resistive target, the general shape of the optimization curves are comparable. Also the number of employed electrode locations is similar, with the optimization on the homogeneous resistivity model usually employing 2-6 electrodes fewer than on the resistive target. A significant difference can only be observed during the first 25 iterations $(<180$ measurements), where the optimization on the homogeneous model uses up to 25 electrodes fewer than for the resistive target, where more electrodes are required to gain improvements.

Figure 8 also shows the relative resolution of the standard survey design, comprising 1591 dipole-dipole and Wenner-Schlumberger configurations, both inline and crossline. As for the comprehensive and optimized surveys, the relative resolution of the standard design on the resistive target is significantly smaller $(S=0.06)$ than on the homogeneous model $(S=0.21)$. 
For the same number of measurements the relative resolution achieved using optimized survey designs is 0.54 and 0.26 for the homogeneous model and resistive target, respectively, increases in resolution of $157 \%$ and $333 \%$. Thus, the optimization survey design calculated on the resistive target should provide better results, given that the underlying assumption of a strong resistivity contrast holds true. Those considerable improvements are achieved using 28 and 26 electrodes fewer than used in the standard survey (homogeneous model and resistive target, respectively), reductions of $24 \%$ and $22 \%$.

Figure 9 shows the distribution of the resolution along a slice through the model domain at $y=$ $0.5 \mathrm{~m}$, both for the standard and optimized surveys. For the homogeneous model (compare Figure 9c against 9a) resolution is improved particularly in deeper parts of the model, while, e.g., at the top of the slope resolution decreases. Within the target volume, the largest absolute increase in resolution can be found at the shallow parts between $x=0.5$ and $0.65 \mathrm{~m}$. While deeper parts show smaller absolute increases in resolution, the increase relative to the standard survey exceed $100 \%$ and are thus higher than for the shallow parts. Similarly to the results obtained on the linear electrode array example, the large weighting exponent of $\beta=5.0$ forces improvements more strongly towards the target volume, while resolution decreases away from it. The reduction is strongest close to the boundaries of the model domain (Figure 9e), where also fewer electrodes are employed. This observation is independent of type of resistivity model used for the optimization, as similar patterns of resolution improvements and reductions can be found for the optimization of the resistive target (Figure 9f). However, improvements relative to the standard survey are considerably higher within the target volume (exceeding 200 $\%$, comparing Figures $9 \mathrm{~b}$ and d), despite the absolute values remaining low. This comparison to the resolution of the standard survey shows that a precise knowledge of the target's locations is not a prerequisite for improved imaging results, as the resolution increases in a wider area around the target employing an optimized survey design. 
421 Figures 9e and falso show the employed electrode locations. The pattern is comparable for the 422 two optimizations. Electrodes along the $x=0.0$, and $y=1.0$ boundaries tend to be rejected by the optimization routine, as well as electrodes on top of the slope. This is somewhat surprising, as for imaging a deeper target, conventional survey designs would usually employ larger electrode spacing. However, measurements with large electrode spacing usually have larger measurement errors and are therefore "penalized" in the optimization. This exercise shows that those outer electrodes are not required to gain high resolution in the target volume, and highlights the potential of this optimization approach to increase the efficiency of ERT imaging, by reducing costs for cables and instrumentation.

The imaging capability of the different survey designs was tested by defining the target volume as a resistive feature $(5000 \Omega \mathrm{m})$ within a $10 \Omega \mathrm{m}$ background material. This may represent, e.g., a clay embankment with a structural defect at its base, which could cause soil piping or slope instabilities, and is of the same order of magnitude as expected for the laboratory experiments. All synthetic data were contaminated with $2 \%$ voltage-dependent noise, and the inversion converged to fit these data within its error levels, using the same inversion parameters as for the linear electrode array example.

The results of the comprehensive set (Figure 10a) resemble the distribution of the resolution (Figure 7); the target is imaged with a strong resistivity contrast between $x=0.4 \mathrm{~m}$ and $0.8 \mathrm{~m}$, showing resistivities above $30 \Omega \mathrm{m}$, with a maximum of $163.5 \Omega \mathrm{m}$. The mean resistivity in the target volume is $33.0 \Omega \mathrm{m}$. This shows the effect of the lower resolution within a resistive target; the difference between the imaged and the true resistivity $(5000 \Omega \mathrm{m})$ is more than one order of magnitude. Its centre is imaged with the highest contrasts, which decrease towards the edges, imaging it with an oval shape. With increasing distance along the $x$-direction, and thus increasing depth from the surface, the target becomes less well resolved, with smaller resistivity contrasts and a shift of the highest values to shallower layers. Thus, the resistive target seems 
446 to have an apparent dip. Between $x=0.8$ and $1.0 \mathrm{~m}$, the resistivity contrast becomes smaller,

447 and therefore could be overprinted by natural resistivity variations in real applications.

448 The standard survey, using about $12.5 \%$ of the measurements of the comprehensive set, images

449 the target with a smaller resistivity contrast than the comprehensive set, having a maximum of

$450 \quad 67.6 \Omega \mathrm{m}$ and a mean resistivity in the target volume of $22.4 \Omega \mathrm{m}$. Considering an iso-volume

451 at $30 \Omega \mathrm{m}$, the target is imaged extending from $x=0.41$ to $0.71 \mathrm{~m}$, while for the comprehensive set, it extend from 0.41 to $0.80 \mathrm{~m}$. Deeper parts of the model, $x>0.70 \mathrm{~m}$, show lower resistivities than imaged with the comprehensive set and the contrast is less sharply defined. The optimized survey designs image the target with a higher resistivity contrast and larger extent than the standard survey, independently of their underlying resistivity model. However, the target is imaged with a higher maximum resistivity $\left(\rho_{\max }=113.8 \Omega \mathrm{m}\right)$ and mean resistivity in the target volume $\left(\rho_{\text {mean }}=28.1 \Omega \mathrm{m}\right)$ when using the resistive target in the optimization, than if using a homogeneous model $\left(\rho_{\max }=100.5 \Omega \mathrm{m}, \rho_{\text {mean }}=26.2 \Omega \mathrm{m}\right)$. Considering again a 30 $\Omega \mathrm{m}$ iso-volume, the target is imaged to extend from 0.40 to $0.73 \mathrm{~m}$ for both optimized surveys.

The improved performance of the optimized surveys is highlighted when looking at the uncentered Pearson correlation coefficient. While for the standard survey a Pearson correlation coefficient of $r_{\text {standard }}=0.29$ is obtained, for the optimized set the correlation is better with $r$ hom $=0.31$ and $r_{\text {resistive }}=0.33$. Thus, in comparison to standard survey designs improved imaging results can be obtained using the optimization methodology, despite requiring up to $24 \%$ fewer electrodes. Even higher reductions in number of electrodes used can be expected for smaller targets.

\section{LABORATORY EXPERIMENT}

469 To test the applicability of the optimization methodology to measured data, a laboratory tank was prepared as described in the previous synthetic example. To ensure a mostly homogeneous 
background medium, a $1 \mathrm{~m} \times 1 \mathrm{~m}$ laboratory tank was filled with pre-prepared, moist

472 (volumetric moisture content $(\mathrm{VMC})$ of $\left.0.31 \mathrm{~m}^{3} / \mathrm{m}^{3}\right)$ pottery clay of low shrinkage $(<5 \%)$, and a mean resistivity of $17 \Omega \mathrm{m}$. The target was constructed using kiln-dried silica sand with a grain size below $0.5 \mathrm{~mm}$ and a $\mathrm{VMC}<0.04 \mathrm{~m}^{3} / \mathrm{m}^{3}$; its resistivity was estimated to be $>5000$ $\Omega m$. Electrode layout and surface topography was as described for the synthetic example. Data were acquired using a Geolog2000 GeoTom system (employing one channel at 8 1/3 Hz) and were measured in normal and reciprocal configurations, where the reciprocal measurement is equivalent to the normal, but with interchanged current and injection dipoles (LaBrecque et al., 1996). The data were defined as the mean of the two measurement, and the error as the standard error in the mean, which is referred to as reciprocal error hereafter. The measurement sequence was reordered to minimize potential polarization effects of the electrodes (Wilkinson et al., 2012). Despite using small electrodes (1.55 $\mathrm{mm}$ diameter, $5 \mathrm{~mm}$ length) contact resistances between electrodes and clay were below $1.1 \mathrm{k} \Omega$. The data quality was very good, with about $99 \%$ of the data having reciprocal errors below 5\%. Analysis of the reciprocal error distribution (Koestel et al., 2008) confirmed the applicability of the previously introduced linear error model (equation 7), but measurement errors were actually lower, so coefficients of $a=0.0025$ $\Omega$ and $b=0.003$ were used to weight the data in the inversion. The data were inverted using E4D (Johnson et al., 2010), employing the same inversion parameters as for synthetic examples. The inversions converged fitting the data to their corresponding error levels, at RMS misfits between modelled and measured data of $2.1-3.2 \%$.

The results are similar to those obtained in the synthetic model, but with the target showing a considerably higher resistivity (Figure 11). Thus, the true target resistivity likely to be higher than was assumed in the synthetic model and used for the calculation of the optimized survey design. This would reflect field usage of this technique where the actual resistivity of the target area is unlikely to be known exactly. The comprehensive survey images the target with a 
maximum resistivity of $969.7 \Omega \mathrm{m}$ and a mean resistivity in the target volume of $108.5 \Omega \mathrm{m}$.

497 The resistive anomaly follows mostly the actual target location, with a slight overestimation in depth for shallow parts $(0.4 \mathrm{~m}<x<0.7 \mathrm{~m})$ and an underestimation in deeper parts $(x>0.8 \mathrm{~m})$. Thus, the target shows an apparent dip as in the previous section. The $60 \Omega \mathrm{m}$ iso-volume highlights this dip, but images the target with a reasonable accuracy (Figure 11a). The standard survey shows the shallow parts of the target at the correct location, but with a smaller resistivity contrast; the maximum resistivity is 264.6 and the mean target resistivity $49.9 \Omega \mathrm{m}$. The apparent dip is more pronounced, as the resistive anomaly bends towards shallower depths and resistivities decrease considerably. The $60 \Omega \mathrm{m}$ iso-volume extends only until $x=0.84 \mathrm{~m}$, and becomes narrow for $x>0.7 \mathrm{~m}$ (Figure 11b). The results for the optimized survey assuming a homogeneous resistivity model show some improvement compared to the standard survey; the target is imaged with a maximum resistivity of $404.0 \Omega \mathrm{m}$ and a mean resistivity of $62.6 \Omega \mathrm{m}$. The narrowing of the $60 \Omega \mathrm{m}$ iso-volume for $x>0.7 \mathrm{~m}$ is less pronounced, but extends only to $x=0.81 \mathrm{~m}$. Better imaging results are achieved using the optimization assuming a resistive target, where the target volume is imaged with a maximum of $490.7 \Omega \mathrm{m}$ and a mean of 68.2 $\Omega \mathrm{m}$. The $60 \Omega \mathrm{m}$ iso-volume is comparable to the one obtained from the comprehensive set. Thus, the resistivity values obtained from the optimized surveys are closer to the

514 Even though the resistivity distribution in the tank can be estimated, variations in degree of compaction of the material and moisture content may cause variations. Therefore, the resistivity model of the comprehensive survey is taken as the imaging benchmark. Considering

517 the uncentered Pearson correlation and the RMS difference highlights the improved performance of the optimized survey designs compared to the standard design. The Pearson correlation coefficient between the imaged resistivities using the comprehensive and standard survey design is $r_{\text {standard }}=0.88$, while for optimization assuming a homogeneous model and a 
resistive target it is $r_{\text {hom }}=0.94$ and $r_{\text {resistive }}=0.96$, respectively. This highlights that if very large

522

resistivity contrasts exist in the subsurface, these should be accounted for in the optimization, as it has a significant effect on the model resolution, as shown in Figures 6 and 8. The RMS differences between the imaged resistivities obtained from the optimized sets and the comprehensive set are $\mathrm{RMS}_{\text {hom }}=32.7 \%$ and $\mathrm{RMS}_{\text {resistive }}=29.8 \%$, while it is $\mathrm{RMS}_{\text {standard }}=$ $40.4 \%$ for the standard survey design. This highlights the considerable improvements that can be achieved when using the proposed optimization methodology, while reducing the amount of required electrode locations.

\section{DISCUSSION AND CONCLUSION}

Optimization of survey design can usually by categorised as (1) trying to find optimum measurement configurations on a given set of electrode locations, or (2) selecting electrode locations based on their comprehensive resolution. This paper presents a modification to the "Compare-R" algorithm, which combines the two approaches by introducing an additional weight penalizing the addition of electrode locations to the optimized set.

Tests on synthetic examples showed that optimization step size and model discretization have negligible effects on the results. Experimenting with different weighting exponents $\beta$, which controls how much the addition of electrodes to the optimized set is penalized, showed that higher values of $\beta$ cause more focussed improvements in resolution and the use of smaller numbers of electrodes, with the drawback of decreasing resolution away from the target volume. Therefore, high values of $\beta$ should be used if the target location and size is well known, and smaller values if it is more uncertain. For an example using a linear electrode array, it was shown that superior resolution compared to a standard survey design can be achieved, despite using $56 \%$ fewer electrodes. To test the impact of the number of electrodes in the comprehensive set, the optimization was run for 16 and 64 electrodes, half and twice the 
number of electrodes in the shown example. While for 16 electrodes, all electrodes are required

to achieve high resolution in the target area, using double the amount of electrodes had no considerable impact on the outcome of the optimization, as electrodes were chosen in the same area as shown for a comprehensive set of 32 electrodes.

549 An investigation of the effect of the mesh discretisation on the calculated sensitivities showed that unstructured tetrahedral meshes can introduce a slight degree of asymmetry into the results.

This is caused by the tetrahedral elements not having the same symmetry as the distribution of electrodes and can be overcome by using a mesh discretization with different polyhedra, such as cuboids.

The methodology was also tested on a 3D synthetic example and verified with a laboratory experiment. The 3D example imaged a structural, highly resistive, defect within a miniaturized embankment model. Here the effect of the underlying resistivity model on the performance of the optimization was tested. By accounting for the resistive target better results were obtained, increasing the uncentered Pearson correlation coefficient between the imaged resistivities and the forward model from $r_{\text {standard }}=0.29$ for the standard survey to $r_{\text {resistive }}=0.33$ for the optimized survey assuming a resistive target. The uncentered Pearson correlation between model and imaged resistivities was $r$ hom $=0.31$ for the optimized survey assuming a homogeneous medium. This is in agreement with previous studies, which showed that small variations in the resistivity distribution have negligible effects on the optimization (e.g., Athanasiou et al., 2006). Here, accounting for a strong resistivity contrast improved the performance of the optimization, but not significantly. Although no marked improvement was obtained, both optimized surveys were able to image the resistive target better than the standard survey design while using fewer electrodes. This was true for the synthetic models and the laboratory experiment. In the latter, accounting for the resistive target helped to increase the Pearson correlation coefficient between optimized and the comprehensive sets from $r_{\text {hom }}=0.94$ to 
$r_{\text {resistive }}=0.96$, which were both superior to the Pearson correlation of the standard survey design $\left(r_{\text {standard }}=0.88\right)$. Here a high weighting factor of $\beta=5.0$ was used, thereby achieving this improved resolution despite using up to $24 \%$ fewer electrodes than a comparable standard survey. Note that using smaller values of $\beta$ would yield higher resolution within the target area, with the cost of using more electrodes. Higher values were tested but reduced the number of electrodes only marginally while causing a further decrease in resolution of the target.

This study shows that by using optimization algorithms that can penalize the number of electrodes used, the efficiency of resistivity imaging and monitoring can be increased by reducing its costs. It may offer the opportunity for high-resolution resistivity imaging using smaller numbers of electrodes and therefore cables, but also using measurement systems capable of addressing only a limited number of electrodes. This may be particularly important for complex monitoring studies with limited accessibility, or where the installation of electrodes may be difficult or detrimental to the structural integrity. For this purpose, values of $\beta>5$ may be chosen to reduce the number of electrodes to a minimum. On the other hand, if measurement time is a priority, smaller values of $\beta$ may be used, allowing to gain high resolution at comparably small measurement sets. We envisage the greatest benefit of the presented approach would be for monitoring or characterisation studies where information about the location of areas of interest are available prior to the survey, e.g., for leaking flood embankments, landslides with well-defined slip surfaces, or contamination studies with reasonably well known hydrology. Additional research is required to implement further constraints on the survey design, such as a priori limitations regarding the maximum number of electrode locations, or pre-defined maximum lengths of survey lines, but also to investigate the practical performance of recently developed measurement configurations (Falco et al., 2013; Szalai et al., 2015, 2014, 2002). Recent research of Loke et al. (2015) shows that calculation times for the optimization can be reduced by assuming symmetry of the 

measurement configurations and exploiting developments in the GPU technology. Comparing 596 their calculation times to the calculation times presented here, GPU and other computational 597 developments may reduce the calculation time of the presented approach by up to 100 times. 598 Smaller calculation times will certainly increase the applicability of the survey optimization 599 and may help to investigate larger-scale problems.

600

\section{ACKNOWLEDGMENTS}

602 We would like to thank the editor (Alexis Maineult), Sándor Szalai, and an anonymous 603 reviewer for their helpful comments on our original manuscript. This paper is published with 604 the permission of the Executive Director of the British Geological Survey (NERC). The 605 developed code and acquired data is archived by the British Geological Survey and can be 606 obtained from the authors. 


\section{REFERENCES}

Alfouzan, F.A., Loke, M.H., Nawawi, M.N.M., 2010. An evaluation of optimization strategies to automatically select the optimal set of array configurations for $2 \mathrm{D}$ electrical imaging surveys. J. Geophys. Eng. 7, 332-342. doi:10.1088/1742-2132/7/3/011

Athanasiou, E., Tsourlos, P., B. Papazachos, C., N. Tsokas, G., 2006. Optimizing Resistivity Array Configurations by Using a Non-Homogeneous Background Model, in: Near Surface 2006. Helsinki, pp. 4-6. doi:10.3997/2214-4609.201402687

Athanasiou, E.N., Tsourlos, P.I., Papazachos, C.B., Tsokas, G.N., 2009. Optimizing Electrical Resistivity Array Configurations by Using a Method Based on the Sensitivity Matrix, in: Near Surface 2009. Dublin, pp. 7-9. doi:10.3997/2214-4609.20147025

Benisch, K., Köhn, D., al Hagrey, S., Rabbel, W., Bauer, S., 2015. A combined seismic and geoelectrical monitoring approach for $\mathrm{CO} 2$ storage using a synthetic field site. Environ. Earth Sci. 73, 3077-3094. doi:10.1007/s12665-014-3603-0

Curtis, A., 1999. Optimal design of focused experiments and surveys. Geophys. J. Int. 139, 205-215. doi:10.1046/j.1365-246X.1999.00947.x

Curtis, A., Maurer, H., 2000. Optimizing the design of geophysical experiments: Is it worthwhile? Lead. Edge 19, 1058-1062. doi:10.1190/1.1438475

Daily, W., Ramirez, A., Binley, A., 2004. Remote Monitoring of Leaks in Storage Tanks using Electrical Resistance Tomography: Application at the Hanford Site. J. Environ. Eng. Geophys. 9, 11-24. doi:10.4133/JEEG9.1.11

Dumont, G., 2017. Geophysical characterization and monitoring of a municipal solid waste landfill. Université de Liège. doi:http://hdl.handle.net/2268/206929

Falco, P., Negro, F., Szalai, S., Milnes, E., 2013. Fracture characterisation using geoelectric null-arrays. J. Appl. Geophys. 93, 33-42. doi:10.1016/j.jappgeo.2013.03.005

Furman, A., Ferré, T.P., Heath, G.L., 2007. Spatial focusing of electrical resistivity surveys considering geologic and hydrologic layering. GEOPHYSICS 72, F65-F73. doi:10.1190/1.2433737

Gance, J., Malet, J.-P., Supper, R., Sailhac, P., Ottowitz, D., Jochum, B., 2016. Permanent electrical resistivity measurements for monitoring water circulation in clayey landslides. J. Appl. Geophys. 126, 98-115. doi:10.1016/j.jappgeo.2016.01.011

Glendinning, S., Hughes, P., Helm, P., Chambers, J., Mendes, J., Gunn, D., Wilkinson, P., Uhlemann, S., 2014. Construction, management and maintenance of embankments used for road and rail infrastructure: implications of weather induced pore water pressures. Acta Geotech. 9, 799-816. doi:10.1007/s11440-014-0324-1

Godio, A., Arato, A., Chiampo, F., Ruggeri, B., Di Addario, M., Fischetti, M., Perissinotto, E., 2015. Liquid injection to enhance biogas production in landfills for pretreated municipal solid wastes - BIO.LEA.R. Project (life+program). Environ. Eng. Manag. J. $14,1623-1636$.

Grellier, S., Guerin, R., Robain, H., Bobachev, A., Vermeersch, F., Tabbagh, A., 2008. Monitoring of Leachate Recirculation in a Bioreactor Landfill by 2-D Electrical Resistivity Imaging. J. Environ. Eng. Geophys. 13, 351-359. doi:10.2113/JEEG13.4.351 
Hilbich, C., Hauck, C., Hoelzle, M., Scherler, M., Schudel, L., Völksch, I., Vonder Mühll, D., Mäusbacher, R., 2008. Monitoring mountain permafrost evolution using electrical resistivity tomography: A 7-year study of seasonal, annual, and long-term variations at Schilthorn, Swiss Alps. J. Geophys. Res. 113, F01S90. doi:10.1029/2007JF000799

Johnson, T.C., 2016. Real-time 4D electrical resistivity imaging of tracer transport within an energically stimulated fracture zone, in: AGU Fall Meeting. San Francisco, USA.

Johnson, T.C., Slater, L.D., Ntarlagiannis, D., Day-Lewis, F.D., Elwaseif, M., 2012. Monitoring groundwater-surface water interaction using time-series and time-frequency analysis of transient three-dimensional electrical resistivity changes. Water Resour. Res. 48, 1-13. doi:10.1029/2012WR011893

Johnson, T.C., Versteeg, R.J., Ward, A., Day-Lewis, F.D., Revil, A., 2010. Improved hydrogeophysical characterization and monitoring through parallel modeling and inversion of time-domain resistivity and induced-polarization data. Geophysics 75, WA27. doi:10.1190/1.3475513

Koestel, J., Kemna, A., Javaux, M., Binley, A., Vereecken, H., 2008. Quantitative imaging of solute transport in an unsaturated and undisturbed soil monolith with 3-D ERT and TDR. Water Resour. Res. 44, n/a-n/a. doi:10.1029/2007WR006755

Krautblatter, M., Verleysdonk, S., Flores-Orozco, A., Kemna, A., 2010. Temperaturecalibrated imaging of seasonal changes in permafrost rock walls by quantitative electrical resistivity tomography (Zugspitze, German/Austrian Alps). J. Geophys. Res. $115,1-15$.

Kuras, O., Wilkinson, P.B., Meldrum, P.I., Oxby, L.S., Uhlemann, S., Chambers, J.E., Binley, A., Graham, J., Smith, N.T., Atherton, N., 2016. Geoelectrical monitoring of simulated subsurface leakage to support high-hazard nuclear decommissioning at the Sellafield Site, UK. Sci. Total Environ. 566-567, 350-359. doi:10.1016/j.scitotenv.2016.04.212

LaBrecque, D.J., Miletto, M., Daily, W., Ramirez, A., Owen, E., 1996. The effects of noise on Occam's inversion of resistivity tomography data. Geophysics 61, 538-548.

Loke, M.H., Chambers, J.E., Rucker, D.F., Kuras, O., Wilkinson, P.B., 2013. Recent developments in the direct-current geoelectrical imaging method. J. Appl. Geophys. 95, 135-156. doi:10.1016/j.jappgeo.2013.02.017

Loke, M.H., Wilkinson, P.B., Chambers, J.E., 2010. Fast computation of optimized electrode arrays for 2D resistivity surveys. Comput. Geosci. 36, 1414-1426. doi:10.1016/j.cageo.2010.03.016

Loke, M.H., Wilkinson, P.B., Chambers, J.E., Uhlemann, S.S., Sorensen, J.P.R., 2015 a. Optimized arrays for 2-D resistivity survey lines with a large number of electrodes. J. Appl. Geophys. 112, 136-146. doi:10.1016/j.jappgeo.2014.11.011

Loke, M.H., Wilkinson, P.B., Tejero-Andrade, A., Kruse, S., 2015b. Optimized Arrays for Resistivity Measurements Confined to the Perimeter of a Survey Area, in: Near Surface Geoscience 2015 - 21st European Meeting of Environmental and Engineering Geophysics. Turin, pp. 6-10. doi:10.3997/2214-4609.201413793

Loke, M.H., Wilkinson, P.B., Uhlemann, S.S., Chambers, J.E., Oxby, L.S., 2014. Computation of optimized arrays for 3-D electrical imaging surveys. Geophys. J. Int. 
Maurer, H., Friedel, S., 2006. Outer-space sensitivities in geoelectrical tomography. Geophysics 71, G93. doi:10.1190/1.2194891

Parsekian, A.D., Singha, K., Minsley, B.J., Holbrook, W.S., Slater, L., 2015. Multiscale geophysical imaging of the critical zone. Rev. Geophys. 53, 1-26. doi:10.1002/2014RG000465

Roux, E., Garcia, X., 2014. Optimizing an experimental design for a CSEM experiment: methodology and synthetic tests. Geophys. J. Int. 197, 135-148. doi:10.1093/gji/ggt525

Rucker, D., 2014. Investigating motion blur and temporal aliasing from time-lapse electrical resistivity. J. Appl. Geophys. 111, 1-13. doi:10.1016/j.jappgeo.2014.09.010

Schmidt-Hattenberger, C., Bergmann, P., Labitzke, T., Wagner, F., Rippe, D., 2016. Permanent crosshole electrical resistivity tomography (ERT) as an established method for the long-term CO 2 monitoring at the Ketzin pilot site. Int. J. Greenh. Gas Control 52, 432-448. doi:10.1016/j.ijggc.2016.07.024

Singha, K., Day-Lewis, F.D., Johnson, T., Slater, L.D., 2014. Advances in interpretation of subsurface processes with time-lapse electrical imaging. Hydrol. Process. 29, n/a-n/a. doi:10.1002/hyp. 10280

Stummer, P., Maurer, H., Green, A.G., 2004. Experimental design: Electrical resistivity data sets that provide optimum subsurface information. GEOPHYSICS 69, 120-139. doi:10.1190/1.1649381

Supper, R., Ottowitz, D., Jochum, B., Kim, J., Römer, A., Baron, I., Pfeiler, S., Lovisolo, M., Gruber, S., Vecchiotti, F., 2014. Geoelectrical monitoring: an innovative method to supplement landslide surveillance and early warning. Near Surf. Geophys. 12, 133-150. doi:10.3997/1873-0604.2013060

Szalai, S., Lemperger, I., Metwaly, M., Kis, Á., Wesztergom, V., Szokoli, K., Novák, A., 2014. Multiplication of the depth of detectability using $\gamma 11 \mathrm{n}$ arrays. J. Appl. Geophys. 107, 195-206. doi:10.1016/j.jappgeo.2014.06.003

Szalai, S., Szarka, L., 2011. Expanding the possibilities of two-dimensional multielectrode systems, with consideration to earlier geoelectric arrays. J. Appl. Geophys. 75, 1-8. doi:10.1016/j.jappgeo.2011.06.020

Szalai, S., Szarka, L., Prácser, E., Bosch, F., Müller, I., Turberg, P., 2002. Geoelectric mapping of near surface karstic fractures by using null arrays. GEOPHYSICS 67, 1769-1778. doi:10.1190/1.1527077

Szalai, S., Wesztergom, V., Szokoli, K., Frigy, A., Prascer, E., 2015. Field Applicability of the g11n Configuration. 8th Congr. Balk. Geophys. Soc. doi:10.3997/22144609.201414151

Tsakirrbaloglou, K., Tsourlos, P., Vargemezis, G., Tsokas, G., 2016. An Algorithm for the Adaptive Optimization of ERT Measurements, in: Near Surface Geoscience 201622ond European Meeting of Environmental and Engineering Geophysics. p. 4. doi:10.3997/2214-4609.201601997

Tsourlos, P., Jochum, B., Supper, R., Ottowitz, D., Kim, J.H., 2016. Optimizing Geoelectrical Arrays for Special Geoelectrical Monitoring Instruments, in: Near Surface Geoscience 
2016- 22ond European Meeting of Environmental and Engineering Geophysics. p. 4. doi:10.3997/2214-4609.201601946

Uhlemann, S., Chambers, J., Wilkinson, P., Maurer, H., Merritt, A., Meldrum, P., Kuras, O., Gunn, D., Smith, A., Dijkstra, T., 2017. Four-dimensional imaging of moisture dynamics during landslide reactivation. J. Geophys. Res. Earth Surf. 122, 398-418. doi:10.1002/2016JF003983

Wagner, F.M., Günther, T., Schmidt-Hattenberger, C., Maurer, H., 2015. Constructive optimization of electrode locations for target-focused resistivity monitoring. GEOPHYSICS 80, E29-E40. doi:10.1190/geo2014-0214.1

Wang, Q., Zhang, X., Zhang, Y., Yi, Q., 2013. AUGEM: Automatically Generate High Performance Dense Linear Algebra Kernels on x86 CPUs, in: Proceedings of the International Conference for High Performance Computing, Networking, Storage and Analysis on - SC '13. ACM Press, New York, New York, USA, pp. 1-12. doi: $10.1145 / 2503210.2503219$

Wilkinson, P.B., Chambers, J.E., Lelliott, M., Wealthall, G.P., Ogilvy, R.D., 2008. Extreme sensitivity of crosshole electrical resistivity tomography measurements to geometric errors. Geophys. J. Int. 173, 49-62. doi:10.1111/j.1365-246X.2008.03725.X

Wilkinson, P.B., Loke, M.H.., Meldrum, P.I., Chambers, J.E., Kuras, O., Gunn, D.A., Ogilvy, R.D., 2012. Practical aspects of applied optimized survey design for electrical resistivity tomography. Geophys. J. Int. 189, 428-440. doi:10.1111/j.1365-246X.2012.05372.x

Wilkinson, P.B., Meldrum, P.I., Chambers, J.E., Kuras, O., Ogilvy, R.D., 2006. Improved strategies for the automatic selection of optimized sets of electrical resistivity tomography measurement configurations. Geophys. J. Int. 167, 1119-1126. doi:10.1111/j.1365-246X.2006.03196.x

Wilkinson, P.B., Uhlemann, S., Meldrum, P.I., Chambers, J.E., Carrière, S., Oxby, L.S., Loke, M.H., 2015. Adaptive time-lapse optimized survey design for electrical resistivity tomography monitoring. Geophys. J. Int. 203, 755-766. doi:10.1093/gji/ggv329 


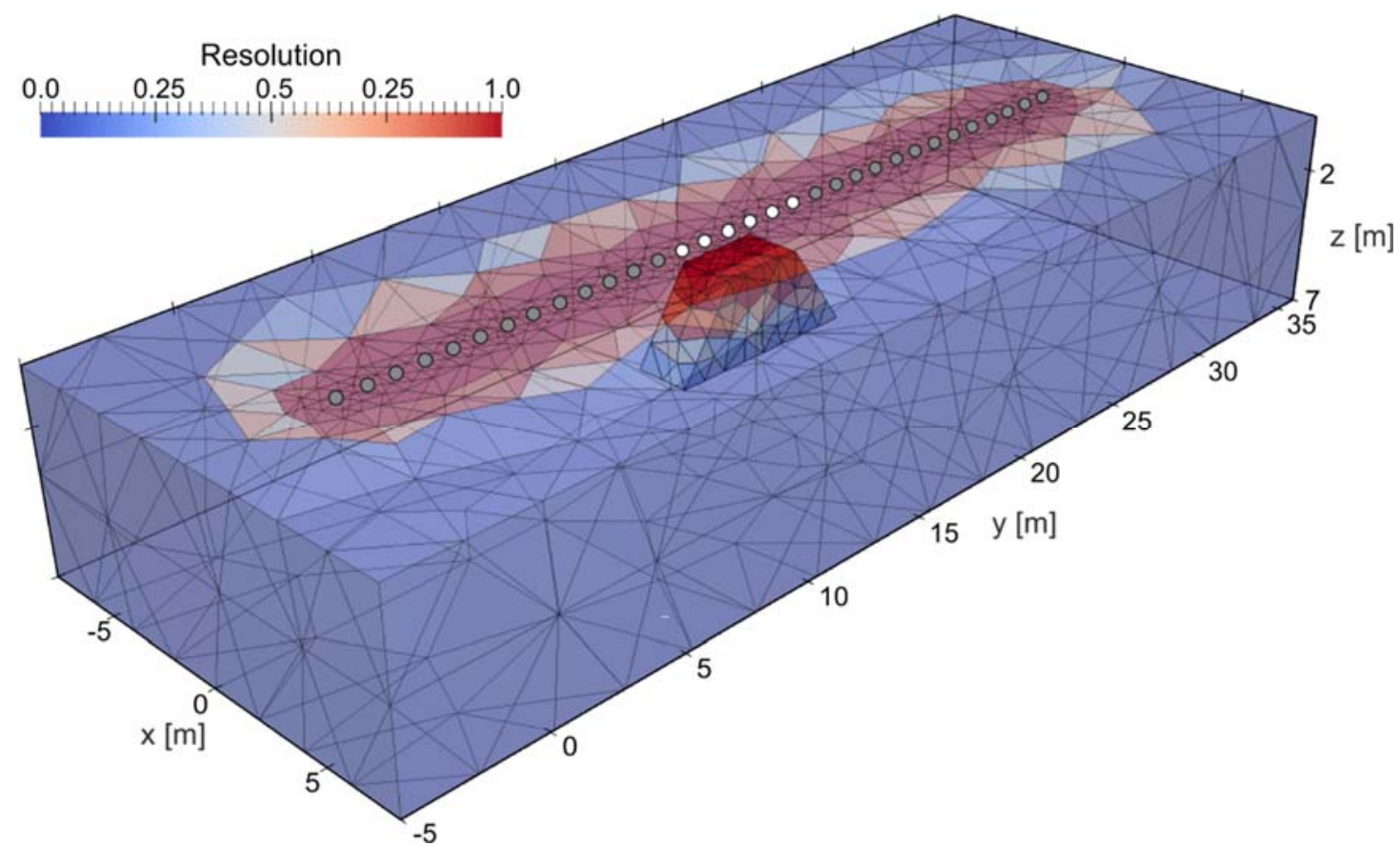

762

763

764

765

766

Figure 1 Comprehensive set model resolution (comprising 70555 measurements); grey lines represent the model discretization. The target volume is shown opaque. Grey dots indicate possible electrode locations; white dots indicate the initial set of electrodes. Note that the slight asymmetry is caused by the discretization of the model.

a)

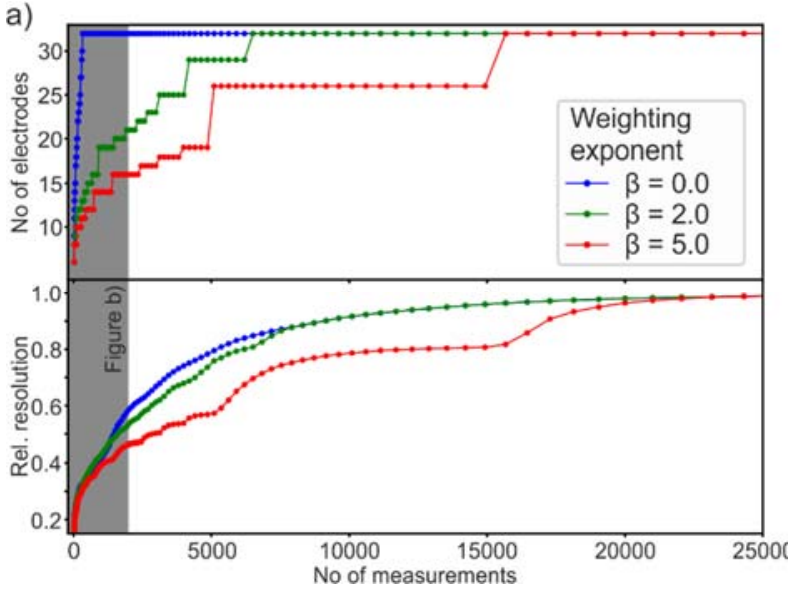

b)

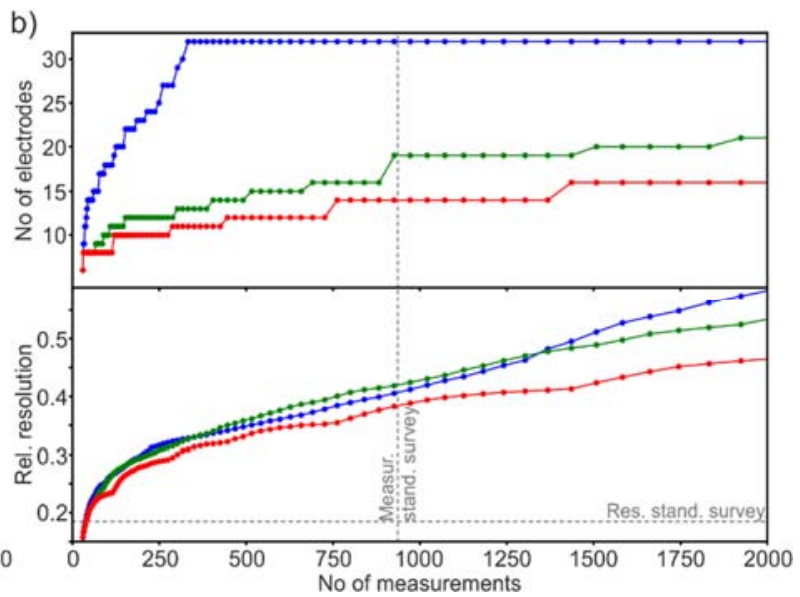

Figure 2 a) Optimization performance in terms of the relative resolution $\mathrm{S}$ and number of used electrodes for weighting exponents $\beta=0.0,2.0$, and 5.0, employing a step size of $5 \%$. The grey area shows the range shown in b). b) subset of a) showing the results for the first 2000 measurements with dashed lines indicating the number of measurements and relative resolution of a standard survey. 

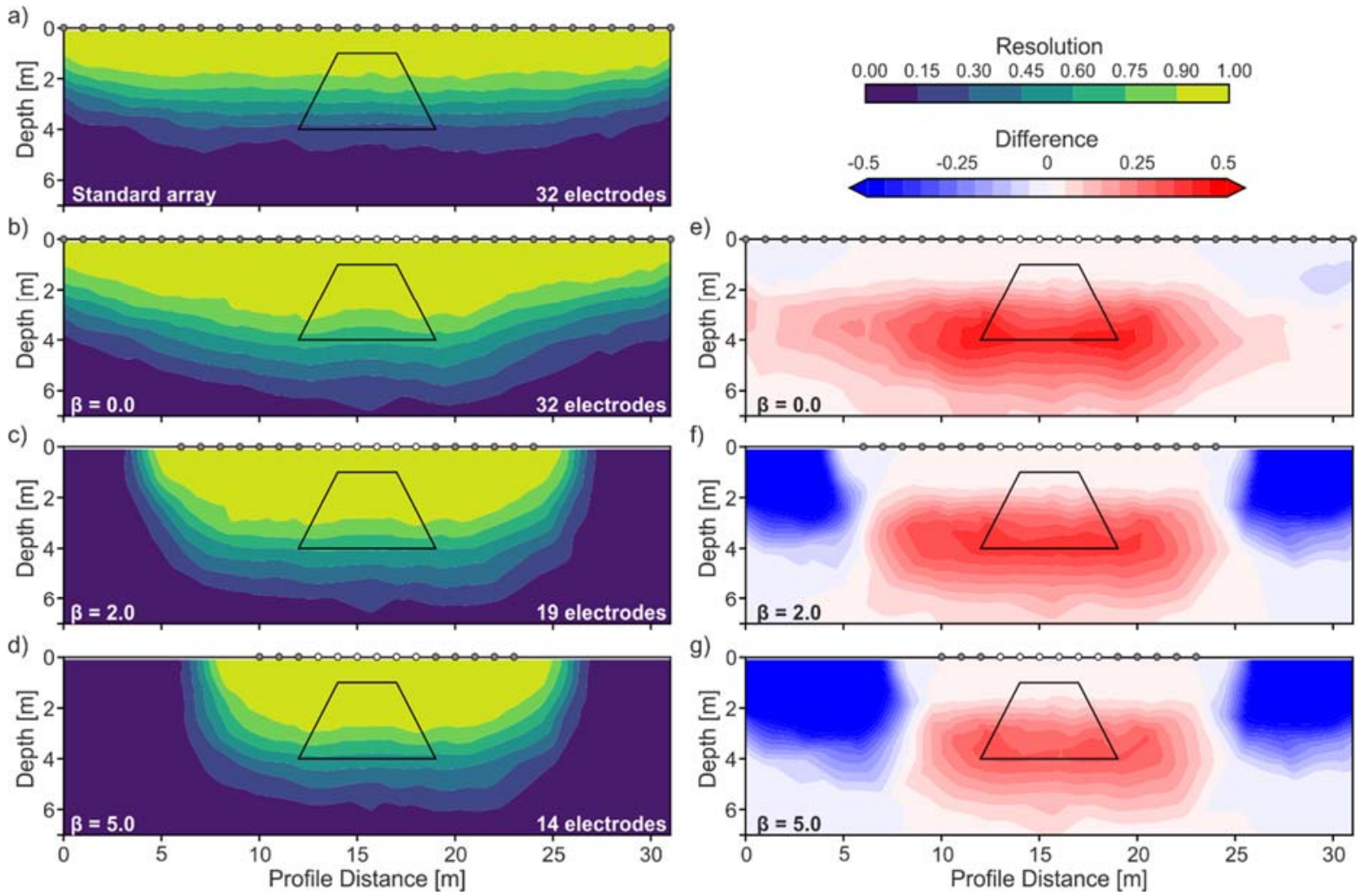

Figure 3 Model resolution for a) standard survey and b)-d) optimized surveys using $\beta=0.0,2.0$, and 5.0. e)-g) difference in model resolution between optimized and standard survey design. In all cases, the resolution within the target volume increased compared to the standard survey. Dots indicate used electrode locations; white dots show the initial set.

a)

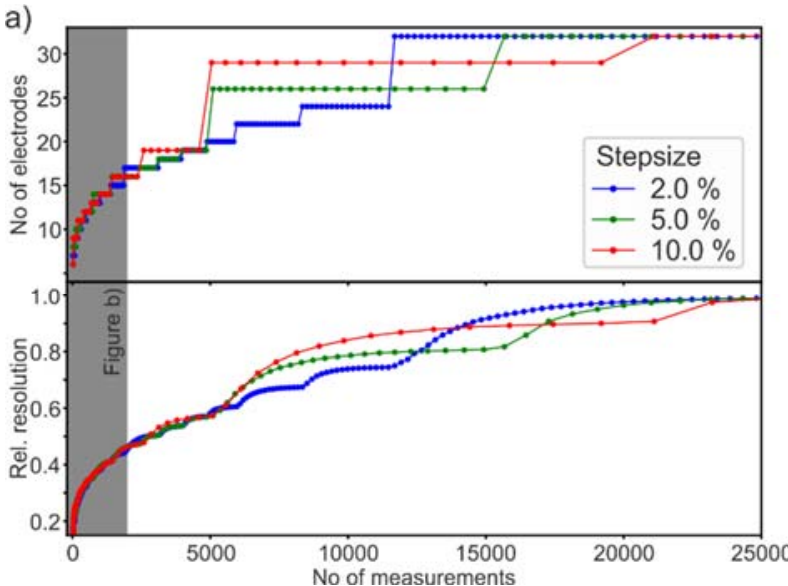

b)

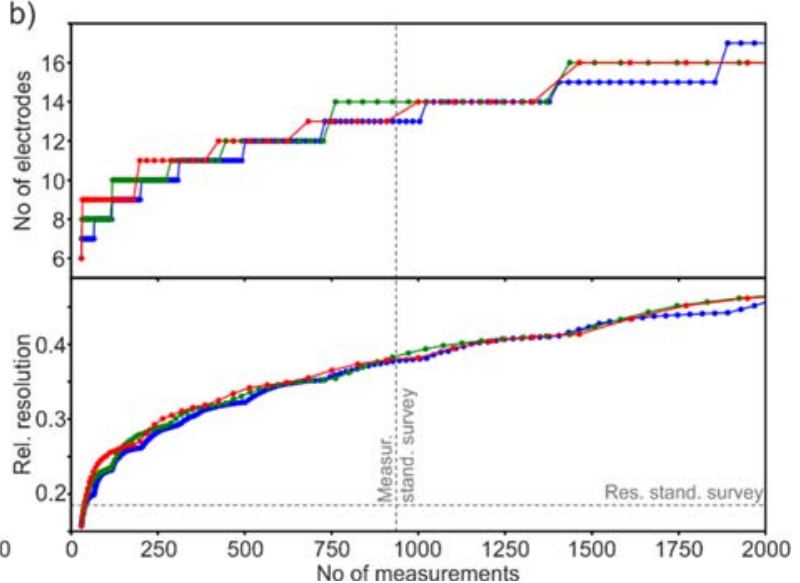

Figure 4 a) Optimization performance in terms of the relative resolution $\mathrm{S}$ and number of used electrodes for $\beta=5.0$ and different step sizes of $2 \%, 5 \%$, and $10 \%$. b) subset of a) showing the results for the first 2000 measurements with dashed lines indicating the number of measurements and relative resolution of a standard survey. 

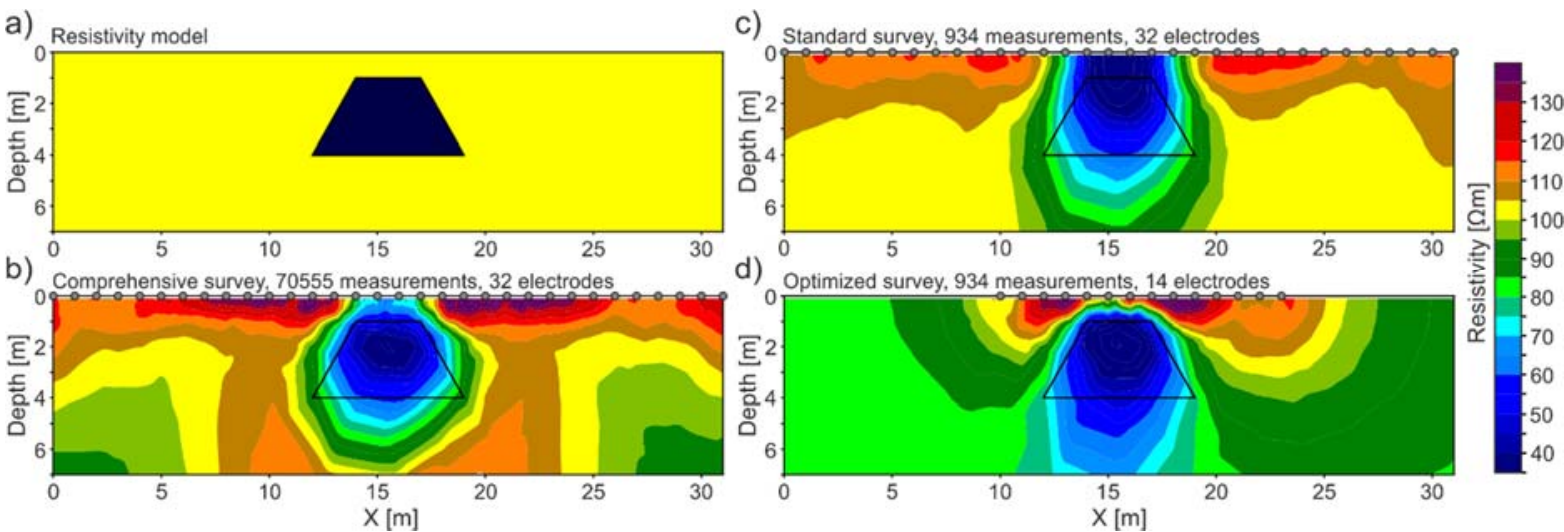

b) Comprehensive survey, 70555 measurements, 32 electrodes

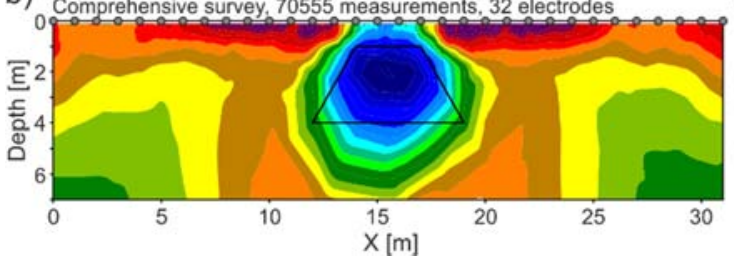

Figure 5 Resistivity models. a) resistivity model employed in the calculation of the synthetic data; b) inverted resistivity model using a comprehensive set of measurements, employing 70555 measurements and 32 electrodes; c) inverted resistivity model using a standard survey design comprising 934 measurements and 32 electrodes; d) inverted resistivity model using an optimized survey comprising 934 measurements, but only 14 electrodes. Note that some asymmetry maybe introduced by the model discretization.

a)

है
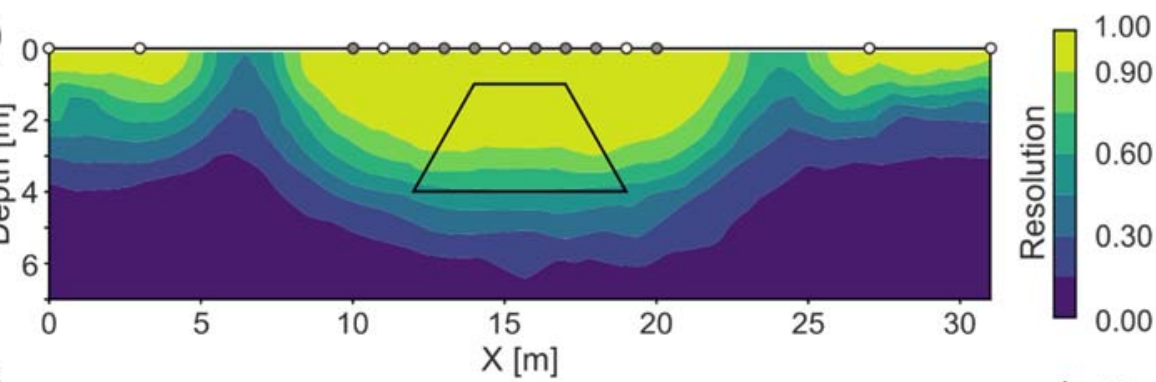

b)
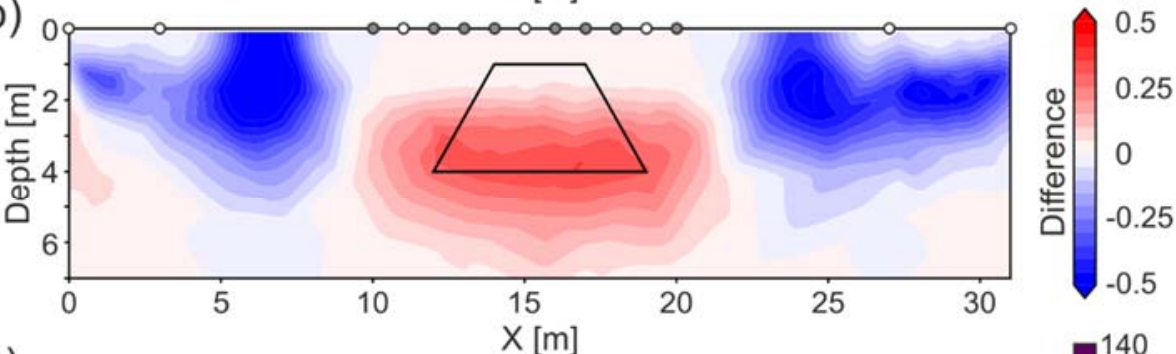

c)

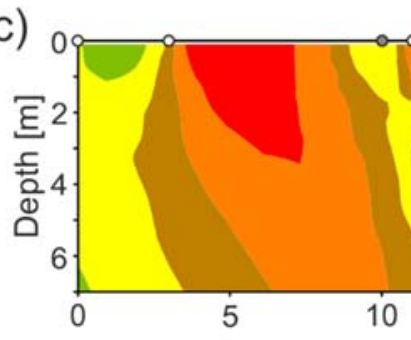

$X[\mathrm{~m}]$

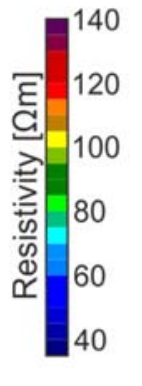

Figure 6 Results for an optimization using an initial set comprising four electrodes close to the section

boundaries and three electrode close to the target. Electrode locations are shown as dots, with white dots indicating the initial locations. a) Resolution of the optimized survey, b) difference in resolution between optimized and standard survey, and c) inverted resistivity model as shown in Figure 5. 


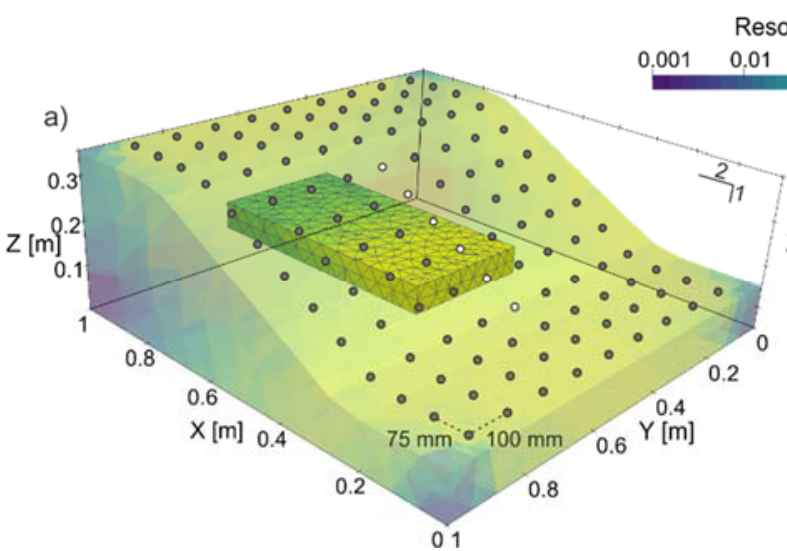

Resolution
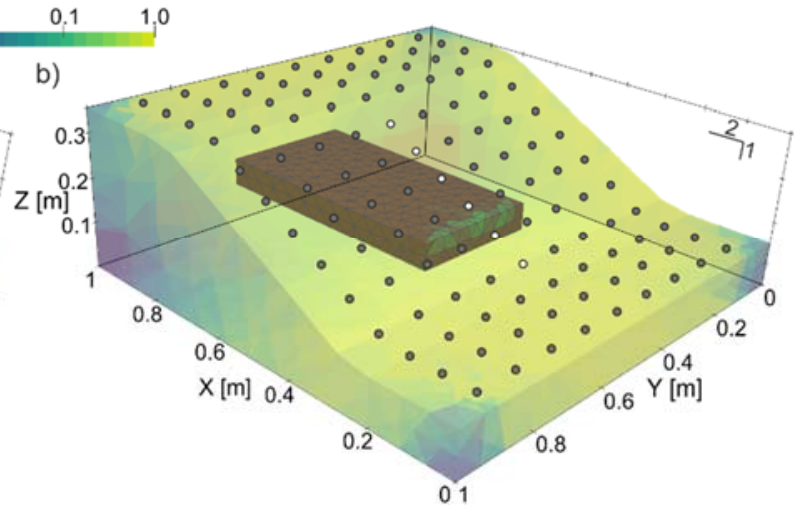

Figure 7 Resolution of the comprehensive set for the synthetic 3D example assuming a homogeneous resistivity distribution of $10 \Omega \mathrm{m}$ (a), and the target as having a resistivity of $5000 \Omega \mathrm{m}$ within a $10 \Omega \mathrm{m}$ background medium (b). The target volume is shown opaque. The dots show the electrode locations, with white dots indicating the initial set of six electrodes.

a)

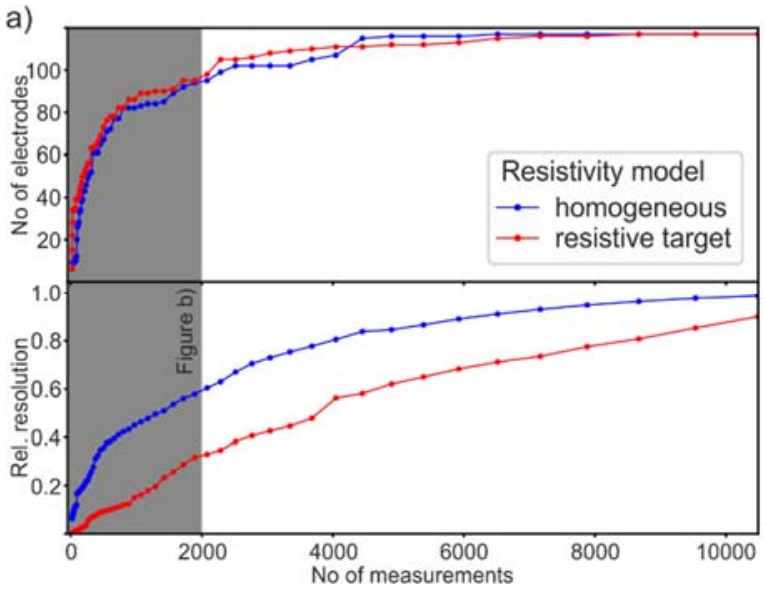

b)

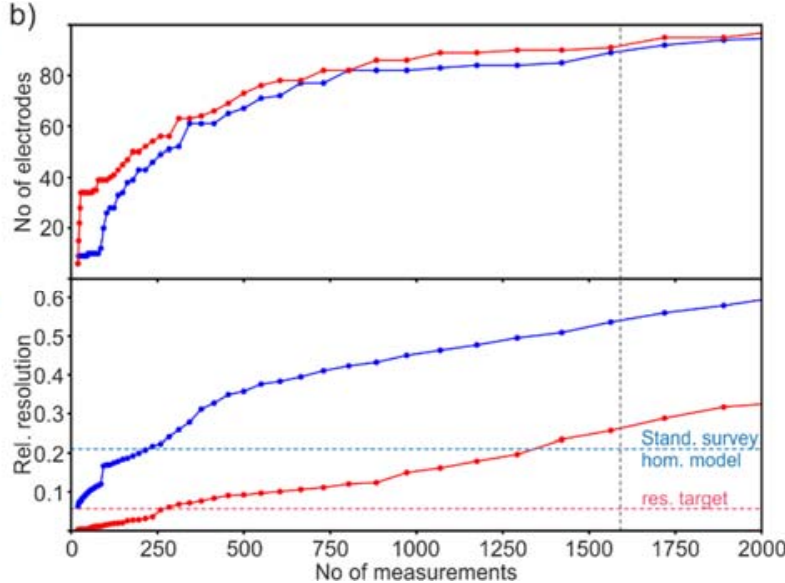

Figure 8 Optimization performance of the 3D example in terms of the relative resolution $\mathrm{S}$ and number of used electrodes for a weighting exponents $\beta=5.0$, employing a step size of $10 \%$, and two different resistivity models. The blue lines show the performance for a homogeneous resistivity model of $10 \Omega \mathrm{m}$, the red lines for a resistive target $(5000 \Omega \mathrm{m})$ within a $10 \Omega \mathrm{m}$ background medium. b) subset of a) showing the results for the first 2000 measurements, with the grey dashed line showing the number of measurements comprising the standard survey design, and the red and blue dashed lines showing the relative resolution of a standard survey on a homogenous and resistive target, respectively. 


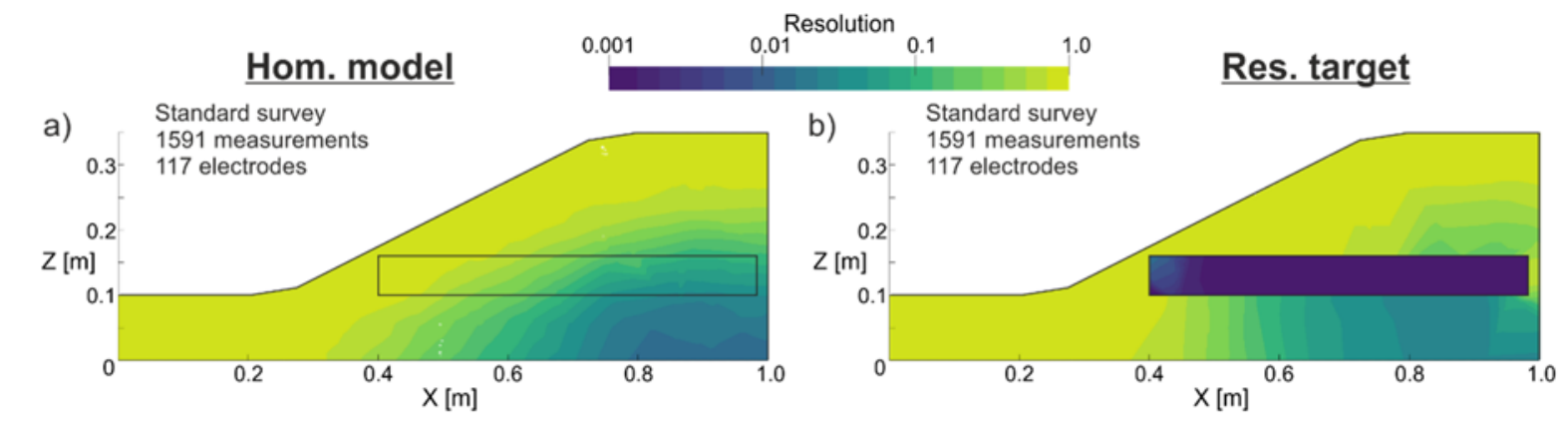

c) $\quad \begin{aligned} & \text { Optimized survey } \\ & 1591 \text { measurements }\end{aligned}$

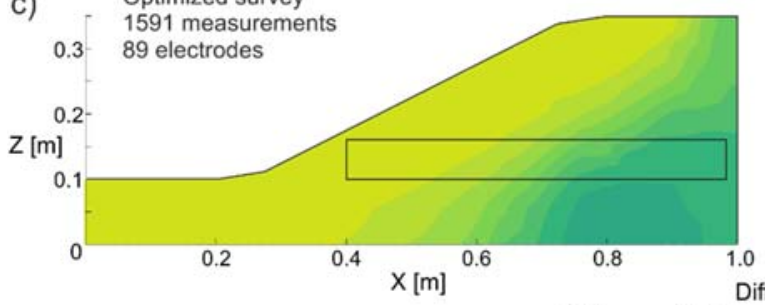

d) $\quad\left[\begin{array}{l}\text { Optimized survey } \\ 1591 \text { measurements }\end{array}\right.$
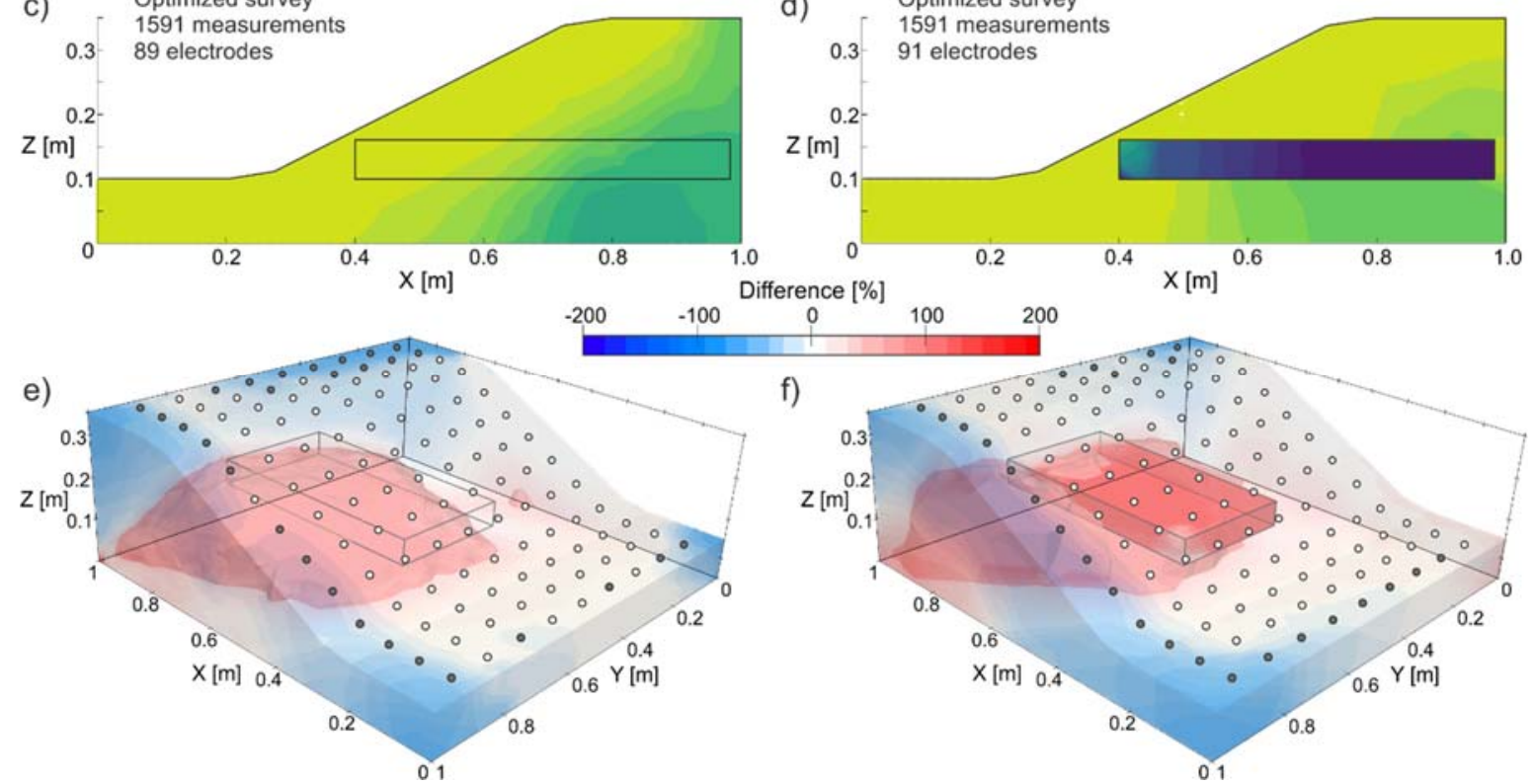

815 Figure 9 Model resolution at $\mathrm{y}=0.5 \mathrm{~m}$ for the standard $(\mathrm{a}, \mathrm{b})$ and optimized survey designs $(\mathrm{c}, \mathrm{d})$, both 816 applied to $(\mathrm{a}, \mathrm{c})$ a homogeneous $(10 \Omega \mathrm{m})$ medium, and $(\mathrm{b}, \mathrm{d})$ with the target being highly resistive $(5000$ $817 \Omega \mathrm{m}$ ). The resolution of this central model domain is clearly improved by the optimized survey designs. 818 The bottom panel (e,f) shows the difference between the optimized and standard survey design, with an iso-volume indicating a $100 \%$ improvement. White dots on the 3D plot indicate used electrode locations, while grey dots show electrode locations which are not used in the optimized design. 

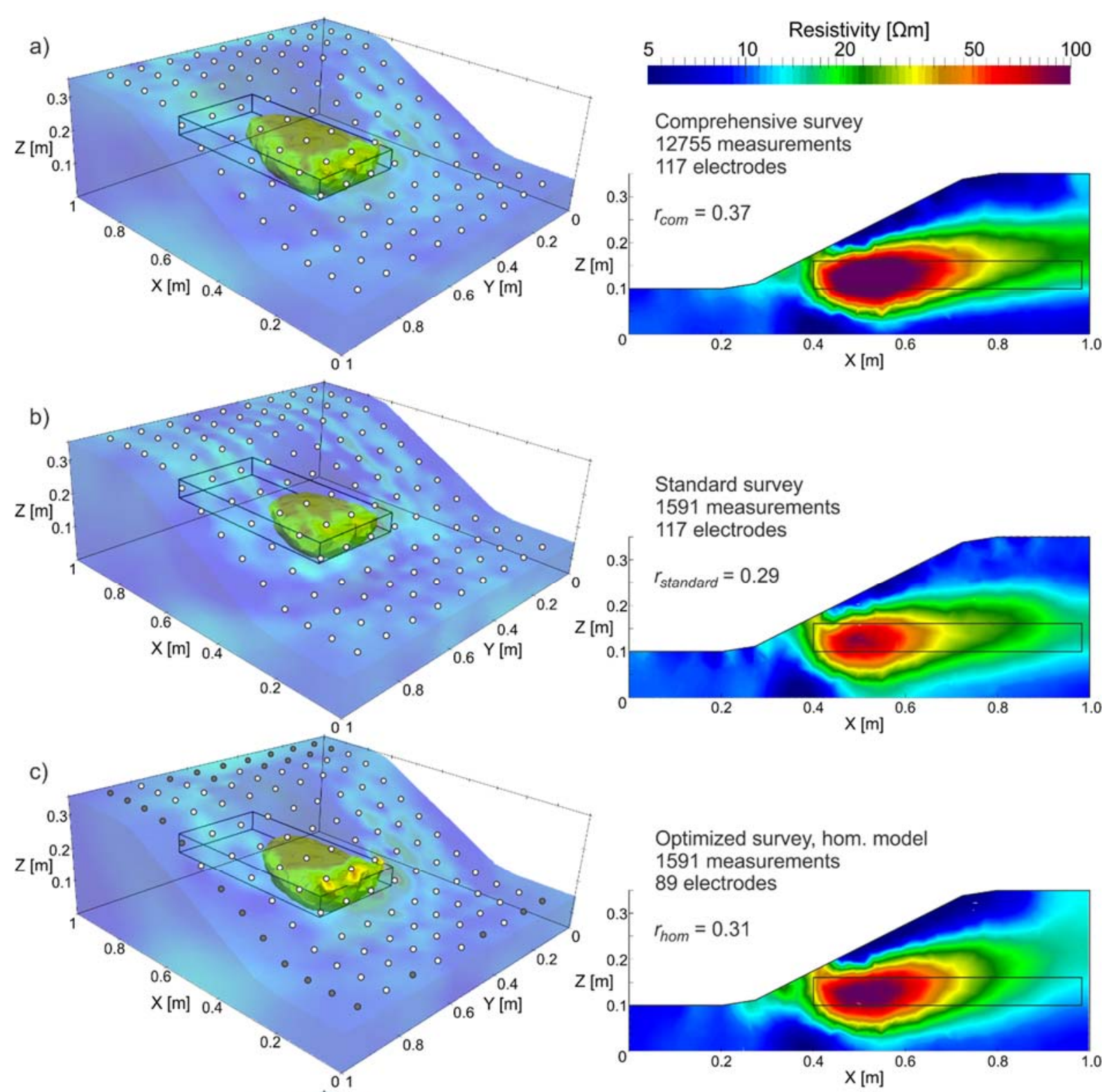

d)
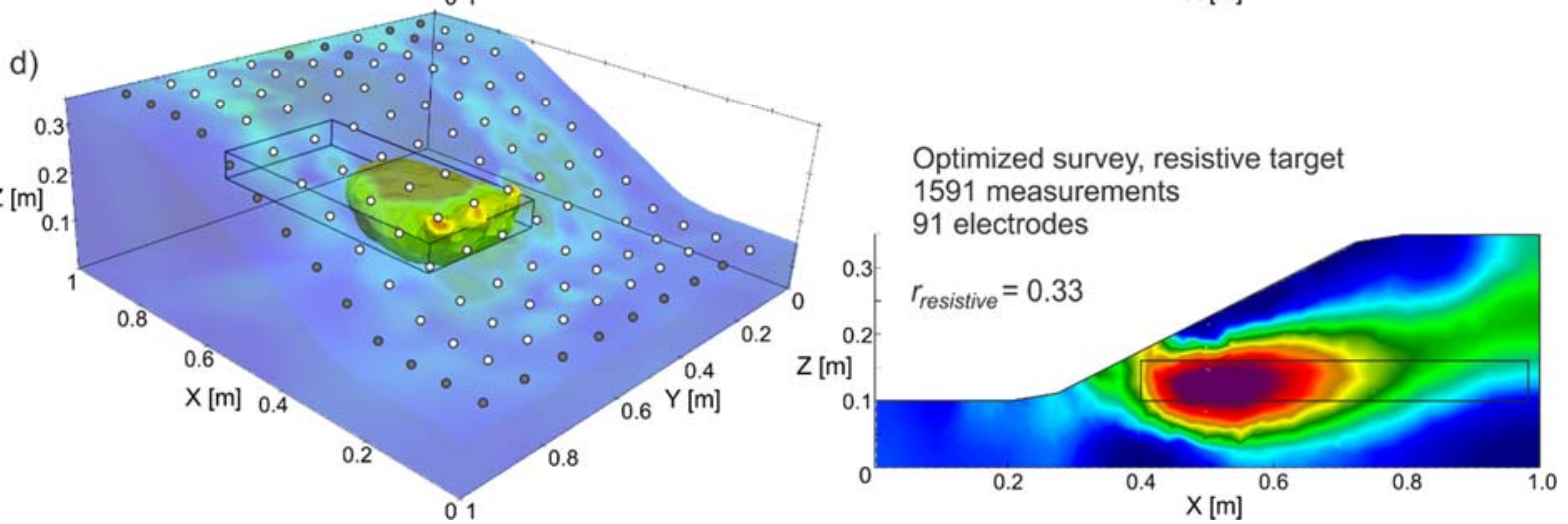

823 Figure 10 Inverted resistivity model for the 3D synthetic example; opaque iso-volumes indicates resistivities $\rho>30 \Omega \mathrm{m}$. a) results for the comprehensive survey, b) for the standard dipole-dipole and Wenner-Schlumberger survey, and c)-d) for the optimized surveys calculated on a homogeneous model and a resistive target, respectively. The slice of the right column is located centrally through the target volume at $y=0.5 \mathrm{~m}$. White dots on the $3 \mathrm{D}$ plots indicate used electrode locations, while grey dots show electrode locations that are not used in the optimized design. 

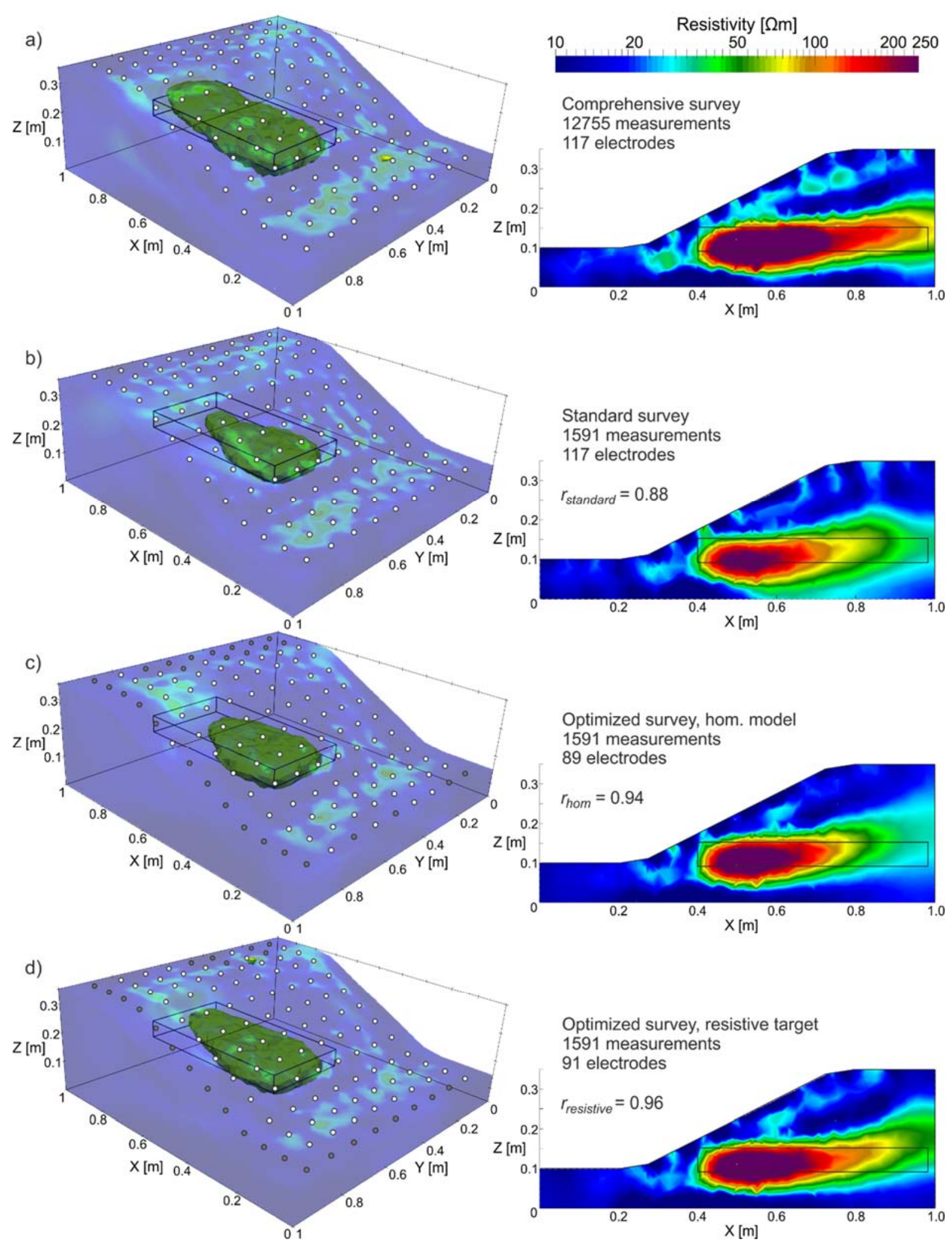

Figure 11 Inverted resistivity models of the laboratory data; opaque iso-volume indicates resistivities of $\rho>60 \Omega \mathrm{m}$; black box outlines the target volume. a) results for the comprehensive survey, b) for the standard dipole-dipole, and Wenner-Schlumberger survey, and c)-d) for the optimized surveys calculated on a homogeneous model and a resistive target, respectively. The slice of the right column is located centrally through the target volume at $y=0.5 \mathrm{~m}$. White dots on the $3 \mathrm{D}$ plots indicate used electrode locations, while grey dots show electrode locations that are not used in the optimized design. 